University of Nebraska - Lincoln

DigitalCommons@University of Nebraska - Lincoln

$6-22-2006$

\title{
An analytical quantum model for intense field processes: Quantum origin of rescattering plateaus
}

M. V. Frolov

Voronezh State University

A. A. Khuskivadze

University of Nebraska - Lincoln

N. L. Manakov

Voronezh State University, manakov@phys.vsu.ru

Anthony F. Starace

University of Nebraska-Lincoln, astarace1@unl.edu

Follow this and additional works at: https://digitalcommons.unl.edu/physicsstarace

Part of the Physics Commons

Frolov, M. V.; Khuskivadze, A. A.; Manakov, N. L.; and Starace, Anthony F., "An analytical quantum model for intense field processes: Quantum origin of rescattering plateaus" (2006). Anthony F. Starace Publications. 124.

https://digitalcommons.unl.edu/physicsstarace/124

This Article is brought to you for free and open access by the Research Papers in Physics and Astronomy at DigitalCommons@University of Nebraska - Lincoln. It has been accepted for inclusion in Anthony F. Starace Publications by an authorized administrator of DigitalCommons@University of Nebraska - Lincoln. 
Published in Journal of Physics B: Atomic, Molecular and Optical Physics 39 (2006), pp. S283-S305; doi 10.1088/0953-4075/39/13/S04 Copyright (C 2006 IOP Publishing Ltd . Used by permission. Online at http://stacks.iop.org/JPhysB/39/S283

Submitted February 23, 2006; published June 22, 2006.

\title{
An analytical quantum model for intense field processes: Quantum origin of rescattering plateaus
}

\author{
M. V. Frolov ${ }^{1}$, A. A. Khuskivadze ${ }^{2,3}$, N. L. Manakov ${ }^{1}$, \\ and Anthony F. Starace ${ }^{2}$ \\ ${ }^{1}$ Department of Physics, Voronezh State University, Voronezh 394006, Russia \\ ${ }^{2}$ Department of Physics and Astronomy, University of Nebraska-Lincoln, Lincoln, \\ NE 68588-0111, USA \\ ${ }^{3}$ Deceased
}

\begin{abstract}
After a brief comparison of two basic quantum approaches for the analytic treatment of strong laser field phenomena, we analyze the spatial and temporal dependences of the quasistationary, quasienergy state wavefunction $\Phi_{\epsilon}(\mathbf{r}, t)$ of a weakly bound level in a strong laser field. We find from this analysis that the characteristic high-energy plateau structures in the spectra of laser- atom processes originate from similar plateau features in the spectra of the Fourierharmonic components, $\Phi_{n}(\mathbf{r})$, of $\Phi_{\epsilon}(\mathbf{r}, t)$. Together with a quantum interpretation of the wellknown rescattering scenario in terms of these wavefunctions, the results of this analysis provide an explanation for the drastic difference between HHG and ATI/ATD in the sensitivity of plateau structures to binding potential effects.
\end{abstract}

\section{Introduction}

Plateau-like structures in the spectra of high-energy electrons or photons emitted by an atomic system subjected to an intense laser field are key features of strong laser-atom processes. The accepted physical interpretation of these features is provided by the rescattering scenario (RS) [1-3], whose basic idea is that even an ionized electron can be "delayed" near the parent atom core by the oscillatory force exerted by the laser field so that the escaping electron can absorb energy during its repeated laser-assisted collisions with the core. A number of qualitative features of plateau structures in strong-field phenomena (such as their extent - or cut-off energy - in both high harmonic generation (HHG) and above-threshold ionization/detachment (ATI/ATD) processes) have been predicted by means of classical or semiclassical considerations based on an analysis of electron trajectories in a laser field (see, e.g., a recent review [4]). 
In addition to its simple physical interpretation of plateau effects, the RS also identifies the major difficulty for the accurate theoretical description of these effects: the necessity for a correct account of electron-core interactions, even in the limit of an intense laser field. For this reason, reliable quantitative information on the rescattering plateaus for a particular atomic or molecular system may only be obtained by direct numerical integration of the time-dependent Schrödinger equation (TDSE). (Note that the first theoretical prediction of the plateau and its cut-off position [for the case of HHG] was based on a numerical solution of the TDSE [5].) By means of such sophisticated calculations, two important facts have been established: (i) plateau effects have a single electron origin and (ii) all qualitative features of plateau structures for real atoms (such as those predicted by recent numerical results for ATI [6] and HHG [7] spectra for the ground state of the hydrogen atom) agree well with results of calculations for short-range binding potentials (with quantitative differences corresponding mainly to the height of the plateau, which may be attributed to Coulomb effects). However, the numerical treatment of the TDSE is a time-consuming task that consequently is performed typically for only a limited set of laser parameters. Furthermore, such completely numerical calculations do not allow one to trace the origin of the characteristic plateau-like structures, the precise role of the RS in their formation and the range of laser parameters over which these features and the mechanisms that create them occur. On the other hand, shortrange potential model systems (which, as noted, give qualitatively accurate predictions of strong-field phenomena) are amenable to detailed analytical analyses starting from a rigorous quantum formulation of the problem. Such analytical analyses may thus be able to reveal the origin of strong-field plateau structures within the framework of a thoroughly quantum treatment.

At the present time, two rather different analytical approaches have been developed for the theoretical description of strong-field processes that include the effect of rescattering on multiphoton transition rates. The $S$-matrix approach (SMA) consists in the straightforward extension to laser-atom problems of the $S$-matrix formalism that has been well developed in formal scattering theory. Introduced by Reiss [8], the SMA was modified to account for rescattering by means of an "improved" Keldysh approximation (IKA) [9]. Through the use of quasiclassical (stationary phase) methods to evaluate transition amplitudes, the IKA provides an effective tool for analysing plateau structures in strong-field phenomena in terms of the RS [4]. Another approach is based on the use of the quasistationary quasienergy states (QQES) formalism for the quantum description of an atomic system in a strong laser field (see, e.g., [10] for a review). The crucial difference between the QQES approach and the SMA is that in the former the problem is formulated in terms of an eigenvalue equation for the complex quasienergy and the corresponding QQES wavefunction, without using the formal evolution operator techniques inherent to the scattering state or to initial value problems. In common with the direct solution of the TDSE, the QQES approach is widely used for direct numerical treatments $[11,12]$. However, for simple short-range potential models, it permits one to also obtain analytical results for the QQES wavefunctions that include an exact account of both laser field and binding potential effects. This approach was applied at first $[13,14]$ to the case of a zero-range potential (ZRP) model system [15] involving a single bound state of s-symmetry that is minimally connected to the three-dimensional continuum (i.e., only through the s-wave scattering phase). This model has been employed for non-perturbative analyses of strong-field effects (including plateau structures) in both ATD and HHG (as has been reviewed elsewhere $[16,17]$ ) as well as in laser-assisted, electron-atom scattering $[18,19]$. Recently [20-22], a more reliable quantum model of strong-field processes has been developed that combines effective range theory [23] for describing a weakly bound sys- 
tem with the QQES approach for describing strong-field interactions with that system. The resulting time-dependent effective range (TDER) theory is largely insensitive to the shape of the short-range binding potential $U(\mathbf{r})$, which supports a weakly bound state having, possibly, non-zero angular momentum (since all information on $U(\mathbf{r})$ in effective range theory is represented by only two parameters, such as, e.g., the scattering length and the effective range [23].) The (one-parameter) ZRP model is a special limiting case. The TDER is most appropriate for describing strong-field interactions with negative ions and for analyzing the dependence of strong-field effects on both the laser parameters and on the spatial symmetry of the initial bound state.

In this paper, after a brief survey of the $S$-matrix formulation of the theory of laser-atom processes, we present a short discussion of the major features and results of the alternative QQES approach as applied to both ZRP and TDER models. Then, using rigorous QQES results for these exactly solvable models, we show how the plateau structures in the highenergy spectra of ATD and HHG may be described in terms of the properties of the exact (model system) wavefunction for a bound electron subjected to a strong monochromatic laser field. In addition, using the exact wavefunction, we demonstrate how the famous Keldysh [24] (or strong-field $[8,25]$ ) approximation (KA) follows from this ab initio quantum treatment. Finally, by introducing the KA wavefunction, which takes into account binding potential effects on the same level as in calculations of the KA ionization amplitude, we are able to show rigorously why the plateau structures in HHG spectra appear already within the KA while those in ATD spectra require a more exact account of binding potential effects for their description.

\section{Brief survey of $S$-matrix and quasienergy approaches for strong-field processes}

We consider the simplest, one-electron model of laser-atom processes, i.e., the electric-dipole interaction, $V(\mathbf{r}, t)$, of a long monochromatic laser pulse (having an electric vector $\mathbf{F}(t)$ $=F \operatorname{Re}[\mathbf{e} \exp (-\mathrm{i} \omega t)]\left(\mathbf{e} \cdot \mathbf{e}^{*}=1\right)$ and an intensity $\left.I=\left(c F^{2}\right) /(8 \pi)\right)$ with an electron that is also subject to a static potential, $U(\mathbf{r})$, that supports both bound and continuum stationary states. The evolution of a bound state, $\psi_{0}(\mathbf{r}, t)=\psi_{0}(\mathbf{r}) \mathrm{e}^{-\mathrm{i} E_{0} t / \hbar}$, or a continuum state, $\psi_{\mathbf{p}}(\mathbf{r}, t)=\psi_{\mathbf{p}}(\mathbf{r})$ $\mathrm{e}^{-\mathrm{i} E t / \hbar}$ (having asymptotic momentum $\mathbf{p}$, where $\mathbf{p}=(2 m E)^{1 / 2}$ ), of an electron subject to both the potential $U(\mathbf{r})$ and the perturbation $V(\mathbf{r}, t)$ is described by the TDSE:

$$
\left(-\frac{\hbar^{2}}{2 m} \nabla^{2}+U(\mathbf{r})+V(\mathbf{r}, t)-\mathrm{i} \hbar \frac{\partial}{\partial t}\right) \Psi(\mathbf{r}, t)=0 .
$$

The solution of equation (1) by means of a non-perturbative treatment of both interactions $U$ and $V$ is the key problem in theoretical studies of intense field processes.

\subsection{The intense field SMA}

For non-perturbative (in $V(\mathbf{r}, t)$ ) calculations of multiphoton transition rates within the framework of the SMA, the transition amplitudes are defined by $S$-matrix elements involving unperturbed (i.e., $\psi_{0}$ or $\left.\psi_{\mathbf{p}}\right)$ states and the corresponding exact solutions $\left(\Psi_{0}\right.$ or $\left.\Psi_{\mathbf{p}}\right)$ of equation (1), i.e., using procedures similar to those involved in the $S$-matrix approach to collisional problems. By means of rather formal manipulations involving Green's functions (or evolution operators) of the total Hamiltonian in (1) and those in which either $U(\mathbf{r})=0$ or $V(\mathbf{r}, t)=0$, the $S$-matrix elements may be expressed in different forms. For example, a commonly used, 
ab initio SMA result for the ionization amplitude of an initial bound state $\psi_{0}(\mathbf{r})$ is $[8,26]$

$$
S_{f i}=-\frac{\mathrm{i}}{\hbar} \int_{-\infty}^{\infty}\left\langle\Psi_{\mathbf{p}}(\mathbf{r}, t)|V(\mathbf{r}, t)| \psi_{0}(\mathbf{r}, t)\right\rangle \mathrm{d} t,
$$

where the brackets $\langle\cdots\rangle$ imply integration over $\mathbf{r}$ and it is assumed that the interaction $V(\mathbf{r}, t)$ turns on at $t=t_{0} \rightarrow-\infty$. The practical implementation of such formal SMA results, like (2), requires one to employ different approximations so as to obtain manageable (though nonperturbative in $V(\mathbf{r}, t)$ ) results for transition rates. The simplest (strong-field) approximation involves the replacement of the exact scattering state $\Psi_{\mathbf{p}}(\mathbf{r}, t)$ in (2) by the free-electron (Volkov) wavefunction in the laser field, $\Psi_{\mathbf{p}}{ }^{V}(\mathbf{r}, t)$. The resulting "Keldysh approximation" (KA) amplitude may be presented in two equivalent forms [4]:

$$
S_{\mathrm{KA}}=-\frac{\mathrm{i}}{\hbar} \int_{-\infty}^{\infty}\left\langle\Psi_{\mathbf{p}}^{V}(\mathbf{r}, t)|V(\mathbf{r}, t)| \psi_{0}(\mathbf{r}, t)\right\rangle \mathrm{d} t=-\frac{\mathrm{i}}{\hbar} \int_{-\infty}^{\infty}\left\langle\Psi_{\mathbf{p}}^{V}(\mathbf{r}, t)|U(\mathbf{r})| \psi_{0}(\mathbf{r}, t)\right\rangle \mathrm{d} t .
$$

The KA amplitude, $S_{\mathrm{KA}}$, however, fails to describe the high-energy (plateau) part of ATI spectra. An improved Keldysh approximation (IKA) amplitude, consisting in taking into account the first order in $U(\mathbf{r})$ corrections to the final state, $\Psi_{\mathbf{p}}{ }^{V}(\mathbf{r}, t)$, in (3), was suggested in [9], where, after some manipulations involving equation (2), the following IKA result for the ionization amplitude was obtained:

$$
S_{\mathrm{IKA}}=-\frac{\mathrm{i}}{\hbar} \int_{-\infty}^{\infty} \int_{-\infty}^{\infty}\left\langle\Psi_{\mathbf{p}}^{V}(\mathbf{r}, t)\left|U(\mathbf{r}) G^{V}\left(\mathbf{r}, t ; \mathbf{r}^{\prime}, t^{\prime}\right) U(\mathbf{r})\right| \psi_{0}\left(\mathbf{r}^{\prime}, t^{\prime}\right)\right\rangle \mathrm{d} t \mathrm{~d} t^{\prime},
$$

where $G^{V}$ is the retarded Green's function for a free electron in the laser field (i.e., the Volkov Green's function, see (20)). It is worthwhile to mention the unusual representation of $S_{\text {IKA }}$ in equation (4) as well as of $S_{\mathrm{KA}}$ in the last formula in (3), i.e. in terms of the atomic potential $U(\mathbf{r})$ instead of the laser-atom interaction $V(\mathbf{r}, t)$. In particular, it creates the (erroneous) impression that the result (4) does not involve the "first order in $U(\mathbf{r})$ " (i.e., KA) amplitude SKA. In section 5.1, we present alternative, "non-SMA" derivations of these results for SKA and SIKA based on our more general definition of the KA, in terms of corresponding wavefunctions.

A quasiclassical analysis of $S_{\text {IKA }}$ (valid for $\hbar \omega \ll\left|E_{0}\right|$ ) involving stationary phase methods for the evaluation of the integrals in (4) yields saddle-point equations that reproduce the basic equations of the RS for ATI [27]. This analysis permits one to explain the basic features of the high-energy plateau and of the angular distributions of ATI electrons [4]. For HHG, the "three-step" RS [28] was confirmed by quasiclassical calculations [29] havingthe same level of accuracy as that of the KA ionization amplitude; i.e., taking into account the binding potential only by means of the initial (bound) state wavefunction, $\psi_{0}(\mathbf{r}, t)$. These results thus represent approximate quantum verifications of the classical RS for both ATI and HHG within the quasiclassical (or low-frequency) approximation. However, even with regard to its general results, such as equation (2), the SMA cannot be regarded as an ab initio quantum approach for the analysis of laser-atom processes. For example, in this approach the light shift of the bound state energy $E_{0}$ in a laser field is neglected even in the lowest order in the intensity (i.e., on the level of the quadratic Stark shift) since these effects obviously cannot be incorporated in the basic result (2) for the ionization amplitude. (Level-shift effects become even more important for the initial state $\psi_{0}$ of an electron having non-zero angular momentum 1 owing to the mixing and splitting of sublevels with different angular momentum projections $m$ by an elliptically polarized laser field.) Furthermore, equation (2) fails to give the correct result for the $n$-photon ionization rate already in the next to the lowest order $\left(\sim I^{n+2}\right)$ of perturbation theory in $V(\mathbf{r}, t)$ since, for the case of a monochromatic perturbation, the level shift terms enter high-order perturbative corrections to the wavefunction [30]. These deficiencies of the intense field SMA are caused by the straightforward application of the stan- 
dard $S$-matrix formalism, which is well-justified mathematically for collision problems (dealing with scattering states), to the case of bound states. For this reason, the intense field SMA cannot be relied upon to reveal the underlying quantum origin of plateau structures (and thus of the RS) in strong-field processes.

The way to incorporate level shift effects of bound states into the $S$-matrix formalism has been formulated by Gell-Mann and Low [31] (see also [32]). In brief, it requires the explicit introduction of an adiabatic factor, $\mathrm{e}^{-\gamma|t|}$, in the perturbation operator. One must then take the limit $\gamma \rightarrow 0$ in the final step of any calculation. This adiabatic $S \gamma(\infty,-\infty)$-matrix approach is commonly used for calculations of transition rates and spectra in the modern theory of multicharged ions (see, e.g., [33]). However, as far as we are aware, it has never been applied for the analysis of laser-atom processes.

\subsection{Quasienergy and complex quasienergy approaches}

The quasienergy state (QES) approach deals with a set of particular solutions of the TDSE (1) having the form

$$
\Psi_{\epsilon}(\mathbf{r}, t)=\mathrm{e}^{-\mathrm{i} \epsilon t / \hbar} \Phi_{\epsilon}(\mathbf{r}, t)=\sum_{s} \Phi_{s}(\mathbf{r}) \mathrm{e}^{-(\mathrm{i} / \hbar)(\epsilon+s \hbar \omega) t},
$$

where the quasienergy (or Floquet) wavefunctions, $\Phi_{\epsilon}(\mathbf{r}, t)$, are (periodic in time) solutions of the Hermitian eigenvalue problem,

$$
\hat{\mathcal{H}}(\mathbf{r}, t) \Phi_{\epsilon}(\mathbf{r}, t) \equiv\left(-\frac{\hbar^{2}}{2 m} \nabla^{2}+U(\mathbf{r})+V(\mathbf{r}, t)-\mathrm{i} \hbar \frac{\partial}{\partial t}\right) \Phi_{\epsilon}(\mathbf{r}, t)=\epsilon \Phi_{\epsilon}(\mathbf{r}, t),
$$

for the quasienergy $\epsilon$, which is a conserved quantum number of an electron in a potential $U(\mathbf{r})$ subjected to a time-periodic interaction satisfying $V(\mathbf{r}, t)=V(\mathbf{r}, t+T)($ where $T=2 \pi / \omega)$ [34]. The quasienergy spectrum of an electron in the combined potential $U(\mathbf{r})+V(\mathbf{r}, t)$ is continuous and the QES wavefunctions $\Phi_{\epsilon}$ form a complete set of scattering states. For such states the usual $S$-matrix methods are valid and equation (6) may be rewritten in the following integral form (the time-dependent generalization of the Lippmann-Schwinger equation for collision problems) :

$$
\Phi_{\epsilon, \mathbf{p}}(\mathbf{r}, t)=\chi_{\epsilon, \mathbf{p}}(\mathbf{r}, t)+\iint G^{V}\left(\mathbf{r}, t ; \mathbf{r}^{\prime}, t^{\prime}\right) U\left(\mathbf{r}^{\prime}\right) \Phi_{\epsilon, \mathbf{p}}\left(\mathbf{r}^{\prime}, t^{\prime}\right) \mathrm{e}^{\mathrm{i} \epsilon\left(t-t^{\prime}\right) / \hbar} \mathrm{d} \mathbf{r}^{\prime} \mathrm{d} t^{\prime},
$$

where $\chi_{\epsilon, \mathbf{p}}(\mathbf{r}, t)$ is the QES solution of (6) with $U(\mathbf{r})=0$, which relates to $\Psi_{\mathbf{p}}{ }^{V}(\mathbf{r}, t)$ as follows: $\Psi_{\mathbf{p}} \mathrm{V}(\mathbf{r}, t)=\chi_{\epsilon, \mathbf{p}}(\mathbf{r}, t) \exp \{-(\mathrm{i} / \hbar) \epsilon t\}$. The quasienergy is $\epsilon=\mathbf{p}^{2} /(2 m)+u_{p}$, where $u_{p}=\left(e^{2} F^{2}\right)$ $/\left(4 m \omega^{2}\right)$ is the ponderomotive energy.

The scattering states $\Phi_{\epsilon, \mathbf{p}}(\mathbf{r}, t)$ are most convenient, e.g., for the analysis of laser-assisted electron scattering (LAES) from the potential $U(\mathbf{r})$. (Approximating $U(\mathbf{r})$ by the ZRP, the analyses of plateau structures in LAES as well as the basic equations of the RS for this process have been performed [18,19] based on the exact solution of Equation 7.) However, for analyses of problems involving an initial bound state, such as ATI and HHG, in not too strong fields (i.e., when the decay of the initial bound state $\psi_{0}(\mathbf{r})$ is exponential in time, so that the concept of a transition rate is applicable), the QQES approach is most appropriate. The QQES wavefunction corresponding to $\psi_{0}(\mathbf{r})$ is also a solution of (6) but satisfies the complex (outgoing wave) boundary condition at $r \rightarrow \infty$ (see (11)). As for quasistationary states in time-independent problems [23], for such an asymptotically divergent solution the operator $\hat{\mathcal{H}}(\mathbf{r}, t)$ in (6) is non-Hermitian so that this solution exists only for a complex value of $\epsilon$, i.e., $\epsilon=\operatorname{Re} \epsilon-\mathrm{i}(\hbar / 2) \Gamma$ where $\Delta \epsilon=\operatorname{Re} \epsilon-E_{0}$ and $\Gamma$ are the energy shift and the total decay 
rate of the state $\psi_{0}(\mathbf{r})$. Alternatively, complex quasienergies and corresponding QQES wavefunctions may also be obtained as the poles and residues of a scattering state wavefunction $\Phi_{\epsilon, \mathbf{p}}(\mathbf{r}, t)$ in the complex $\epsilon$-plane [10]. QQES wavefunctions satisfy an integral equation similar to (7) [35]

$$
\Phi_{\epsilon}(\mathbf{r}, t)=\iint G^{V}\left(\mathbf{r}, t ; \mathbf{r}^{\prime}, t^{\prime}\right) U\left(\mathbf{r}^{\prime}\right) \Phi_{\epsilon}\left(\mathbf{r}^{\prime}, t^{\prime}\right) \mathrm{e}^{\mathrm{i} \epsilon\left(t-t^{\prime}\right) / \hbar} \mathrm{d} \mathbf{r}^{\prime} \mathrm{d} t^{\prime}
$$

but which does not involve an incident wave. Equation (8) is the integral counterpart of the differential eigenvalue equation (6) for the discrete spectrum of complex quasienergies $\epsilon$.

Using properly normalized QQES wavefunctions, one can develop an accurate quantum formulation for the calculation of $n$-photon ATI and HHG rates that take into account the shift and broadening of the initial state $\psi_{0}(\mathbf{r})$ by the laser field. The normalization of QQES wavefunctions requires the introduction of so-called dual functions, $\tilde{\Phi}_{\epsilon}(\mathbf{r}, t)$, in accordance with the normalization condition:

$$
\frac{1}{T} \int_{0}^{T}\left\langle\tilde{\Phi}_{\epsilon}(\mathbf{r}, t) \mid \Phi_{\epsilon}(\mathbf{r}, t)\right\rangle \mathrm{d} t=1
$$

The choice of $\tilde{\Phi}_{\epsilon}(\mathbf{r}, t)$ depends on the laser polarization [11,16]; e.g., for the case of linear polarization, $\tilde{\Phi}_{\epsilon}(\mathbf{r}, t)=\left.\Phi_{\epsilon}(\mathbf{r},-t)^{*}\right|_{m \rightarrow-m}$, where $m$ is the magnetic quantum number of the initial bound state $\psi_{0}(\mathbf{r})$. The dual function should also be used in calculations of matrix elements as the bra vector. Thus, HHG rates $\left(\mathcal{R}_{N}\right)$ are determined by the Fourier components $\tilde{\mathbf{d}}_{N}$ of the "dual" dipole moment matrix element $\mathbf{d}(t)$ corresponding to the harmonic frequency $\Omega$ (where $\Omega=N \omega$ and $N$ is an odd integer) $[16,36]$ :

$$
\mathcal{R}_{N}=\frac{(N \omega)^{3}}{2 \pi \hbar c^{3}} \sum_{m}\left|\tilde{\mathbf{d}}_{N}\right|^{2}, \quad \tilde{\mathbf{d}}_{N}=\frac{1}{T} \int_{0}^{T}\left\langle\tilde{\Phi}_{\epsilon}(\mathbf{r}, t)|\mathbf{d}| \Phi_{\epsilon}(\mathbf{r}, t)\right\rangle \mathrm{e}^{\mathrm{i} N \omega t} \mathrm{~d} t,
$$

where $\mathbf{d}=e \mathbf{r}$. Equations (10) are basic results for analyses of HHG spectra using the QQES method.

The ATI amplitude $\mathcal{A}_{n}$ may be extracted from the asymptotic form of the normalized QQES wavefunction $\Phi_{\epsilon}(\mathbf{r}, t)$ as follows [17,37] (the periodic in $t$ phase factor before the sum in (11) depends on the gauge of $V(\mathbf{r}, t)$ : the expression (11) is written in the length gauge; in the velocity gauge the term $\sim\left(|e| / \omega^{2}\right)(\mathrm{r} \cdot \dot{\mathbf{F}}(t))$ should be omitted) :

$$
\lim _{r \rightarrow \infty} \Phi_{\epsilon}(\mathbf{r}, t)=\exp \left\{\frac{\mathrm{i}}{\hbar}\left[\frac{e^{2}}{2 m \omega^{4}} \int^{t}\left[\dot{\mathbf{F}}\left(t^{\prime}\right)\right]^{2} \mathrm{~d} t^{\prime}-u_{p} t+\frac{|e| \mathbf{r} \cdot \dot{\mathbf{F}}(t)}{\omega^{2}}\right]\right\} \sum_{n} \mathcal{A}_{n} \frac{\mathrm{e}^{\mathrm{i} k_{n} R-\mathrm{i} n \omega t}}{R}
$$

where $\mathbf{R}=\mathbf{r}-\frac{|e|}{m \omega^{2}} \mathbf{F}(t), \dot{\mathbf{F}}(t)=\frac{\partial \mathbf{F}(t)}{\partial t}, \hbar k_{n}=\sqrt{2 m\left(n \hbar \omega+\epsilon-u_{p}\right)}$. The square root in $k_{n}$ is chosen so that in the open ionization channels $\left(\operatorname{Re} k_{n}{ }^{2}>0\right)$, the QQES wavefunction describes outgoing spherical waves; while in the closed channels $\left(\operatorname{Re} k_{n}{ }^{2}<0\right)$, it describes exponentially damped waves.

\section{Basic equations of the TDER theory}

In general, for real atoms an accurate solution of equation (6) for either the QES or the QQES wavefunction is as difficult as the direct solution of the initial value problem presented by the TDSE (1) [11, 12]. However, these functions may be found analytically in the TDER approach [20], i.e., for a potential $U(\mathbf{r})$ that acts only within a short-range sphere, $r<r_{c}$, and supports a shallow bound state, $\psi_{0}(\mathbf{r})=\varphi_{\kappa l m}(r) Y_{l, m}(\hat{\mathbf{r}})$, having an energy $E_{0}=-\hbar^{2} \kappa^{2}(2 m)$ ${ }^{-1}\left(\kappa r_{c} \ll 1\right)$ and an angular momentum $l$. The key simplification in the solution of $(6)$ for this 
case is that the boundary condition for $\Phi_{\epsilon}(\mathbf{r}, t)$ at small $r\left(r \sim r_{c}\right)$ may be expressed in a general form independent of the shape of $U(r)$ (see $\lceil 20,21\rceil$ for details) :

$$
\begin{aligned}
\Phi_{\epsilon}(\mathbf{r}, t) & =\sum_{n} \Phi_{n}(\mathbf{r}) \mathrm{e}^{-\mathrm{i} n \omega t} \\
& =Y_{l, m}(\hat{\mathbf{r}}) \sum_{n}\left(r^{-l-1}+\cdots+B_{l}(\epsilon+\hbar n \omega)\left(r^{l}+\cdots\right)\right) f_{n} \mathrm{e}^{-\mathrm{i} n \omega t},
\end{aligned}
$$

where $f_{n}$ are Fourier coefficients of a periodic function $f_{\epsilon}(t)=\Sigma_{s} f_{S} \exp (-\mathrm{is} \omega t)$, and where the effective range parametrization [23] is used for the coefficient $B_{l}$ :

$$
(2 l-1) ! !(2 l+1) ! ! B_{l}(E)=-1 / a_{l}+r_{l} k^{2} / 2, \quad k^{2}=2 m E / \hbar^{2},
$$

where $a_{l}$ is the scattering length and $r_{l}$ is the effective range. For bound state problems, $a_{l}$ and $r_{l}$ may be expressed in terms of the binding energy $\left|E_{0}\right|$ (or $\left.\kappa=\left(2 m\left|E_{0}\right|\right)^{1 / 2} / \hbar\right)$ and the coefficient $C_{\kappa l}$ in the known asymptotic form of $\varphi_{\kappa l m}(r)$ for any finite-range potential $U(r)$,

$$
\varphi_{\kappa l m}\left(r \gg \kappa^{-1}\right) \approx C_{\kappa l} r^{-1} \exp (-\kappa r),
$$

as follows [38]:

$$
(-1)^{l} \kappa^{2 l+1}-a_{l}^{-1}-r_{l} \kappa^{2} / 2=0, \quad(-1)^{l}(2 l+1)-r_{l} \kappa^{-(2 l-1)}=2 \kappa C_{\kappa l}{ }^{-2} .
$$

Since $U(\mathbf{r})=0$ for $r>r_{c}$, in this domain the general solution of (6) satisfying outgoing wave boundary conditions at $\mathrm{r} \rightarrow \infty$ may be expressed as a wave packet of Volkov states as follows $[20,21]$ :

$$
\Phi_{\epsilon}(\mathbf{r}, t)=-\frac{2 \pi \hbar^{2}}{m} \lim _{\mathbf{r}^{\prime}=0} \mathcal{Y}_{l m}\left(\nabla_{\mathbf{r}^{\prime}}\right) \int_{-\infty}^{t} G^{(V)}\left(\mathbf{r}, t ; \mathbf{r}^{\prime}, t^{\prime}\right) f_{\epsilon}\left(t^{\prime}\right) \mathrm{e}^{\mathrm{i} \epsilon\left(t-t^{\prime}\right) / \hbar} \mathrm{d} t^{\prime},
$$

where $\mathcal{Y}_{l m}\left(\nabla_{\mathbf{r}^{\prime}}\right)$ is the differential operator having the form of a solid harmonic, $\mathcal{Y}_{l m}(\mathbf{r}) \equiv$ $r^{l} Y_{l m}(\hat{\mathbf{r}})$, with the substitution $\mathbf{r} \rightarrow \nabla_{\mathbf{r}}$. The action of this operator on the Volkov Green's function $G^{(V)}\left(\mathbf{r}, t ; \mathbf{r}^{\prime}, t^{\prime}\right)$ ensures the same behavior of the $l$ th partial wave component of the QQES $\Phi_{\epsilon}(\mathbf{r}, t)$ at small $r$ as in the boundary condition (12). Thus, matching (12) and the $l$ wave component of (15) on a sphere of radius $r \sim r_{c}$ yields a one-dimensional, homogeneous integro-differential equation for $f_{\epsilon}(t)$ that in fact replaces the eigenvalue equations (6) and (8) for the complex quasienergy $\epsilon$. This reduction of the four-dimensional equations (6) and (8) to only a one-dimensional eigenvalue problem for $\epsilon$ and $f_{\epsilon}(t)$ is the key advantage of the TDER approach. As an example, the equation for $\epsilon$ and $f_{\epsilon}(t)$ for the case of an s-state $\psi_{0}(\mathbf{r})$ is

$$
\left(E_{0}+\frac{\kappa r_{0}}{2} \Delta\right) f_{\epsilon}(t)+\mathrm{i} \hbar \frac{\kappa r_{0}}{2} \frac{\mathrm{d} f_{\epsilon}(t)}{\mathrm{d} t}=\sqrt{\frac{\hbar\left|E_{0}\right|}{4 \pi \mathrm{i}}} \int_{0}^{\infty} \frac{\mathrm{d} \tau}{\tau^{3 / 2}}\left\{\mathrm{e}^{(\mathrm{i} / \hbar)[\epsilon \tau+S(t, t-\tau)]} f_{\epsilon}(t-\tau)-f_{\epsilon}(t)\right\},
$$

where $\Delta=\epsilon-E_{0}$ and $S(t, t-\tau) \equiv S\left(\mathbf{r}=0, t ; \mathbf{r}^{\prime}=0, t-\tau\right)$ is the classical action for an electron in a laser field $\mathbf{F}(t)$. For numerical treatments, it is convenient to rewrite (16) equivalently as an infinite system of linear homogeneous equations for $\epsilon$ and for the Fourier coefficients, $f_{2 k}$, of $f_{\epsilon}(t)$. (The corresponding equations may be found in $[14,16]$ for an s-state $\psi_{0}(\mathbf{r})$ in the ZRP model; and in $[20,21]$ for a $\mathrm{p}$-state $\psi_{0}(\mathbf{r})$ in the TDER.)

The ZRP model is a special case of the more general TDER treatment. For an s-state and $r_{0}=0$, the boundary condition (12) reduces to that for a ZRP model $[13,14]$,

$$
\Phi_{\epsilon}(\mathbf{r}, t)=Y_{00}(\hat{\mathbf{r}})\left(\frac{1}{r}-\kappa\right) f_{\epsilon}^{\mathrm{zrp}}(t),
$$

where this condition may be formulated at the origin, $r \rightarrow 0$. Correspondingly, the asymptotic form (14) for $\varphi_{\kappa 00}(r)$, with $C_{\kappa 0}=(2 \kappa)^{1 / 2}$ becomes valid over the entire interval $0<r<\infty$, and equation (16) evaluated at $r_{0}=0$ easily reduces to that for $f_{\epsilon}^{\operatorname{zrp}}(t)$ [14]. 


\section{Plateau features in the QQES wavefunction}

\subsection{General remarks}

With the known $\epsilon$ and Fourier coefficients $f_{s}$ of $f_{\epsilon}(t)$ (which in general may be obtained only numerically), the spatial and temporal dependences of the QQES wavefunction (15) may be written explicitly as

$$
\begin{aligned}
& \Phi_{\epsilon}(\mathbf{r}, t)=\sum_{n} \Phi_{n}(\mathbf{r}) \mathrm{e}^{-\mathrm{i} n \omega t} \\
& =-\frac{2 \pi \hbar^{2}}{m} \sum_{s} f_{s} \mathrm{e}^{-\mathrm{i} s \omega t} \lim _{\mathbf{r}^{\prime}=0} \mathcal{Y}_{l m}\left(\nabla_{\mathbf{r}^{\prime}}\right) \int_{-\infty}^{t} G^{V}\left(\mathbf{r}, t ; \mathbf{r}^{\prime}, t^{\prime}\right) \exp \left(\mathrm{i}(\epsilon+s \hbar \omega)\left(t-t^{\prime}\right) / \hbar\right) \mathrm{d} t^{\prime},
\end{aligned}
$$

in which the expression for $G^{V}\left(\mathbf{r}, t ; \mathbf{r}^{\prime}, t^{\prime}\right)$ is known. In particular, for the case of a linearly polarized field $(\mathbf{F}(t)=\hat{z} F \cos \omega t)$, to which we restrict our considerations in the rest of this paper, the Green's function $G^{V}$ using the length gauge for $V(\mathbf{r}, t)$ is

$$
G\left(\mathbf{r}, t ; \mathbf{r}^{\prime}, t^{\prime}\right)=-\frac{\mathrm{i}}{\hbar}\left(\frac{m}{2 \pi \mathrm{i} \hbar\left(t-t^{\prime}\right)}\right)^{3 / 2} \Theta\left(t-t^{\prime}\right) \exp \left(\frac{\mathrm{i}}{\hbar} S\left(\mathbf{r}, t ; \mathbf{r}^{\prime}, t^{\prime}\right)\right),
$$

where $\Theta(x)$ is the Heaviside function,

$$
\begin{aligned}
S\left(\mathbf{r}, t ; \mathbf{r}^{\prime}, t^{\prime}\right)= & \frac{m\left(\mathbf{r}-\mathbf{r}^{\prime}-\alpha(t)+\alpha\left(t^{\prime}\right)\right)^{2}}{2\left(t-t^{\prime}\right)}-u_{p}\left(t-t^{\prime}\right) \\
& +\frac{u_{p}}{\omega} \cos \left(\omega\left(t+t^{\prime}\right)\right) \sin \left(\omega\left(t-t^{\prime}\right)\right)+m \mathbf{r} \cdot \dot{\alpha}(t)-m \mathbf{r}^{\prime} \cdot \dot{\alpha}\left(t^{\prime}\right),
\end{aligned}
$$

where

$$
\alpha(t)=\frac{|e| \mathbf{F}(t)}{m \omega^{2}}, \quad \dot{\alpha}(t)=\frac{|e| \dot{\mathbf{F}}(t)}{m \omega^{2}} .
$$

Before discussing the qualitative behavior of the QES harmonics $\Phi_{n}(\mathbf{r})$ in (18) as functions of $n$ and $\mathbf{r}$, we must emphasize the decisive role of the function $f_{\epsilon}(t)$ for understanding the effects of the binding potential $U(\mathbf{r})$ in strong-field processes. As may be seen from equation (12), the coefficients $f_{n}$ determine the population of QES harmonics $\Phi_{n}(\mathbf{r})$ at small distances, $r \sim r_{c}$. These harmonics appear as the result of absorption $(n>0)$ or stimulated emission $(n<0)$ of $|n|$ photons by an (initially bound) electron localized near the origin and having a fixed angular momentum $l$. According to the effective range theory [23], in this $l$ wave channel the short-range potential $U(r)$ affects the electron scattering phase shift $\delta_{l}(k)$. Therefore, in the TDER model, only the electron in the $l$-wave channel "feels" the potential $U(r)$ and consequently may absorb and emit photons, thus generating the quasienergy harmonics $\Phi_{n}(\mathbf{r})$ at the origin. As a result, all binding potential effects, which influence the further propagation of the electron in the laser field outside the potential well $U(\mathbf{r})$, are concentrated within the factors $f_{s}$ in (19). Although these coefficients have been introduced initially (at small $r$ ) only in the $l$-wave channel, after matching equation (12) with the function $\Phi_{\epsilon}(\mathbf{r}, t)$ outside the well, they enter the laser-field-induced angular momentum components of $\Phi_{n}(\mathbf{r})$ with $l^{\prime} \neq l$ as well as the quasienergy harmonics $\Phi_{n^{\prime}}(\mathbf{r})$ with $n^{\prime} \neq n$. Thus, they provide the interchannel couplings between the multiphoton channels with different $n$ and $l$ in the QQES wavefunction (18). These couplings stem from the effects of electron interaction with the potential $U(\mathbf{r})$ in the presence of a strong laser field and, in the TDER theory, this interaction is taken into account essentially exactly, on an equal footing with the interaction $V(\mathbf{r}, t)$. 
It is important to note that only even coefficients, $f_{n} \equiv f_{2 k}$, in (12)(and thus $f_{s}$ in (19)) are non-zero for bound state problems. This result is a particular case of a more general relation between the spatial parity of a QES harmonic $\Phi_{n}(\mathbf{r})$ and its number $n$ :

$$
\Phi_{n}(-\mathbf{r})=(-1)^{l+n} \Phi_{n}(\mathbf{r}),
$$

where $l$ is the angular momentum of the initial bound state $\psi_{0}(\mathbf{r})$. This relation follows from (i) selection rules for dipole transitions, according to which absorption or emission of a single photon changes the electron angular momentum by unity, and (ii) from the fact that the parity of the zero-order harmonic $\Phi_{0}(\mathbf{r})$ is the same as for the bound state $\psi_{0}(\mathbf{r})$, i.e., $(-1)^{l}$. Thus, for small $r$ (at which the $l$-wave component in the wavefunction $\Phi_{\epsilon}(\mathbf{r}, t)$ is dominant (cf. (12)) ), multiphoton exchanges between the electron and the laser field must necessarily involve only even numbers of photons.

Since the explicit form of $\Phi_{\epsilon}(\mathbf{r}, t)$ depends on the gauge (by means of the time-dependent factor $\exp \left\{\mathbf{i}|e| \mathbf{r} \cdot \dot{\mathbf{F}}(t) /\left(\hbar \omega^{2}\right)\right\} ;$ see footnote 7$)$, in order to visualize the gauge-independent features in the dependence of $\Phi_{\epsilon}$ on $\mathbf{r}$ and $t$, we introduce the gauge-invariant factor,

$$
\mathcal{N}(\mathbf{r}, t)=\tilde{\Phi}_{\epsilon}^{*}(\mathbf{r}, t) \Phi_{\epsilon}(\mathbf{r}, t)=\sum_{k} \mathcal{N}_{k}(\mathbf{r}) \mathrm{e}^{-\mathrm{i} k \omega t},
$$

where $\tilde{\Phi}_{\epsilon}(\mathbf{r}, t)$ is the dual function (cf. (9)), and where the Fourier coefficient $\mathcal{N}_{k}(\mathbf{r})$ is the following combination of QES harmonics:

$$
\mathcal{N}_{k}(\mathbf{r})=\sum_{n} \tilde{\Phi}_{n}(\mathbf{r}) \Phi_{n+k}(\mathbf{r}),
$$

where $\mathcal{N}_{k}(\mathbf{r})=\mathcal{N}_{-k}(\mathbf{r})$. Equation (9) gives the normalization condition for $\mathcal{N}_{0}(\mathbf{r})$ :

$$
\int \mathcal{N}_{0}(\mathbf{r}) \mathrm{d} \mathbf{r}=\int \sum_{n} \tilde{\Phi}_{n}(\mathbf{r}) \Phi_{n}(\mathbf{r}) \mathrm{d} \mathbf{r}=1 .
$$

The important "parity relation" for $\mathcal{N}_{k}(\mathbf{r})$

$$
\mathcal{N}_{k}(-\mathbf{r})=(-1){ }^{k} \mathcal{N}_{k}(\mathbf{r})
$$

follows from (22). Note that in the QQES approach only bilinear combinations of QQES wavefunctions such as those in (23) enter the transition amplitudes, such as the one in (10).

Concerning the behavior of $\mathcal{N}_{k}(\mathbf{r})$ at small and large $r$, we note that for $r \leq \kappa^{-1}$ the zeroorder QES harmonic, $\Phi_{0}(\mathbf{r})$, is the dominant one among the set of harmonics $\Phi_{n}(\mathbf{r})$ (because $\Phi_{0}(\mathbf{r}) \approx \psi_{0}(\mathbf{r})$ in this region). Thus, as we have confirmed numerically, the following approximate expression for $\mathcal{N}_{k}(\mathbf{r})$,

$$
\mathcal{N}_{k}(\mathbf{r}) \approx \Phi_{0}(\mathbf{r})\left[\Phi_{k}(\mathbf{r})+\tilde{\Phi}_{-k}(\mathbf{r})\right]
$$

has high accuracy. Owing to the time-dependent phase in (11), the asymptotic behavior of the QES harmonic $\Phi_{s}(\mathbf{r})$ at large $r$ cannot be represented by a single outgoing wave; rather, it has the following form:

$$
\lim _{r \rightarrow \infty} \Phi_{s}(\mathbf{r})=\sum_{n} A_{s n} \frac{\mathrm{e}^{\mathrm{i} k_{n} r}}{r},
$$

where the sum over $n$ runs over all open ionization channels and $A_{s n}$ is a linear superposition of $n$-photon ionization amplitudes $\mathcal{A}_{n}$ [37]. We note the following "sum rule":

$$
\sum_{n}\left|\mathcal{A}_{n}\right|^{2}=\sum_{s, n}\left|A_{s n}\right|^{2}
$$

where the sums over $n$ and $s$ involve only open ionization channels. (Although the coefficients $A_{s n}$ in (28) are gauge dependent, the sum over $s$ in (29) is gauge invariant.) Thus, the 

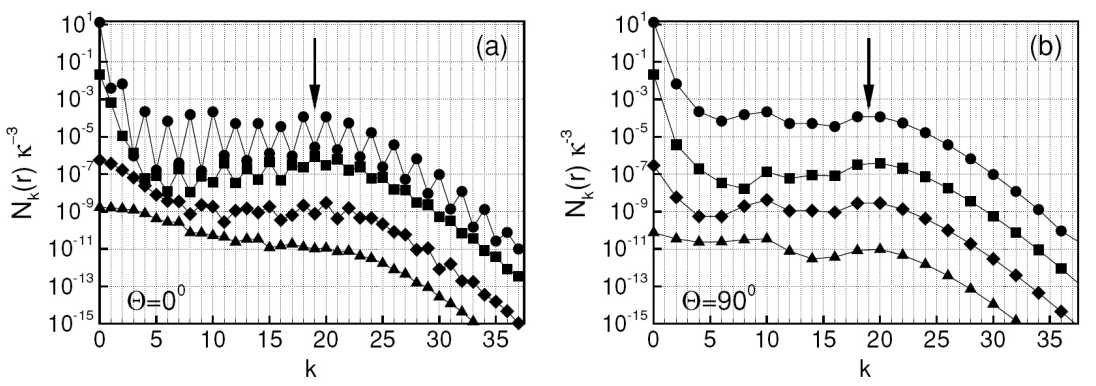

Figure 1. $k$-dependence of the factors $\mathcal{N}_{k}(\mathbf{r})$ for several values of $r$. a) $\Theta=0^{\circ}$; and $\left.\mathbf{b}\right) \Theta=$ 90 . Circles: $r=0.1 \kappa^{-1}$; squares: $r=\kappa^{-1}$; diamonds: $r=5 \kappa^{-1}$; triangles: $r=10 \kappa^{-1}$. The arrow shows the position $k_{\max }=\left(\left|E_{0}\right|+3.17 u_{p}\right) /(\hbar \omega)$. The laser parameters are $\hbar \omega=0.128\left|E_{0}\right|$ and $F=0.12 F_{0}$, where $F_{0}=\left(2 m\left|E_{0}\right|^{3}\right)^{1 / 2} /(|e| \hbar)$.
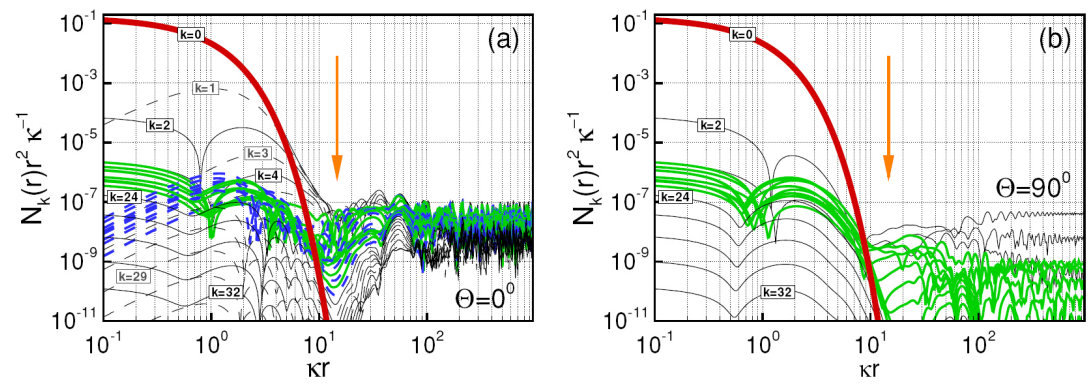

Figure 2. $r$-dependence of the factors $\mathcal{N}_{k}(\mathbf{r}) r^{2}$ for $0 \leq k \leq 32$. a) $\Theta=0^{\circ}$; and b) $\Theta=90^{\circ}$. Harmonics with even (odd) $k$ that do not belong to the "plateau" region are marked by thin solid (thin dashed) lines; harmonics with even (odd) k that do belong to the "plateau" region are marked by thick solid (thick dashed) lines (see the text for details). The arrow shows the position of $r=\alpha_{0}=|e| F /\left(m \omega^{2}\right)=14.65 \kappa^{-1}$. The laser parameters $F$ and $\omega$ are the same as in Figure 1 . The thick solid line for $k=0$ corresponds to the approximation $\mathcal{N}_{0}(\mathbf{r}) \approx \psi_{0}^{2}(\mathbf{r})$.

general expression for the ATD rates $\Gamma_{n}$ may also be expressed directly in terms of the coefficients $A_{s n}$

$$
\Gamma_{n}=\frac{\hbar}{m}\left|\sqrt{k_{n}} \mathcal{A}_{n}\right|^{2}=\frac{\hbar}{m} \sum_{s}\left|\sqrt{k_{n}} A_{s n}\right|^{2}
$$

\subsection{Exact numerical results for $\mathcal{N}_{k}(\mathbf{r})$}

In Figures 1 and 2, we illustrate the behavior of the factors $\mathcal{N}_{k}(\mathbf{r})$ as functions of $k$ and $r$ for the case of a linearly polarized field $\mathbf{F}(t)$ and two directions of the vector $\mathbf{r}$, i.e., along the direction of the laser polarization $\left(\Theta=0^{\circ}\right)$ and in the plane orthogonal to it $\left(\Theta=90^{\circ}\right.$; note that $\mathcal{N}_{k}(\mathbf{r})$ is axially symmetric in this plane). For the sake of simplicity, we present these results for the case of an s-state, $r_{0}=0$ (i.e., for the ZRP model), so that they are scaled by only one parameter, $\kappa=1 / a_{0}$. The spectra of $\mathcal{N}_{k}(\mathbf{r})$ for four values of $r$ are presented in Figure 1. For all $r$ and both directions of $\hat{\mathbf{r}}\left(\Theta=0^{\circ}\right.$ and $\left.90^{\circ}\right)$, well-developed plateau structures with cut-off positions near $k \approx k_{\max }=\left(\left|E_{0}\right|+3.17 u_{p}\right) /(\hbar \omega)$ are clearly visible. For even $k$, the existence of plateau features in $\mathcal{N}_{k}(\mathbf{r})$ near the origin (at small $r$ ) is not surprising, since they are caused 
by similar plateaus (with the same $k_{\max }$ ) in the spectrum of coefficients $f_{2 \mathrm{~s}}[17,19]$. The odd QES harmonics $\Phi_{k}(\mathbf{r})$ are laser-field induced; they are suppressed as compared to the even ones for small values of $r$ (cf. the "saw-tooth" behavior of the curve for $r=0.1 \kappa^{-1}$ in Figure 1(a)), while the difference in magnitudes of $\mathcal{N}_{k}(\mathbf{r})$ for even and odd $k$ disappears with increasing $r$. For $\Theta=90^{\circ}$, only even QES harmonics in Figure 1(b) are non-zero and their magnitude decreases with increasing $r$ in approximately the same fashion as for $\Theta=0^{\circ}$. This fact agrees with the general relation (26) : spherical harmonics $Y_{l m}(\Theta, \varphi)$ with odd $l$ are zero at $\Theta$ $=90^{\circ}$, so that $\mathcal{N}_{2 k+1}(\mathbf{r})=0$ if the vector $\mathbf{r}$ is orthogonal to the axis of laser polarization (or to the polarization ellipse for the case of elliptic polarization).

The $r$-dependence of harmonics $\mathcal{N}_{k}(\mathbf{r})$ with $\mathrm{k} \leq 32$ is shown in Figure 2 for $\Theta=0^{\circ}$ and $\Theta=$ $90^{\circ}$. As may be seen, harmonics $\mathcal{N}_{k}(\mathbf{r})$ for $6 \leq k \leq 23$ nearly coincide to form the plateau-like structure in the interval $0<r<\alpha_{0}$, where $\alpha_{0}$ is the amplitude of free-electron oscillations in a laser field: $\alpha_{0}=|e| F /\left(m \omega^{2}\right)$. For both parallel $\left(\Theta=0^{\circ}\right)$ and orthogonal geometry $\left(\Theta=90^{\circ}\right)$, the basic harmonic $\mathcal{N}_{0}(\mathbf{r})$ is well described by its field-free limit, $\mathcal{N}_{0}(\mathbf{r}) \approx \psi_{0}{ }^{2}(\mathbf{r})$. The relatively smooth, plateau-like dependence of $\mathcal{N}_{k}(\mathbf{r})$ on $r$ exists only inside a sphere of radius $r \approx \alpha_{0}$. Outside this sphere, the QES harmonics $\Phi_{n}(\mathbf{r})$ are drastically modified by the laser field, exhibit highly oscillatory behavior and for $r>10 \alpha_{0}$ their $r$-dependence is completely defined by the asymptotic form (28). To confirm this fact, we have calculated $\Phi_{\epsilon}(\mathbf{r}, t)$ as the sum of $\Phi_{n}(\mathbf{r})$ in (18) for $r=100 \kappa^{-1} \approx 7 \alpha_{0}$ and $r=500 \kappa^{-1} \approx 35 \alpha_{0}$ and then fitted the results to expression (11) by using the least squares $\left(\chi^{2}\right)$ method to find the amplitudes $\mathcal{A}_{n}$, which is equivalent to the calculation of $\Gamma_{n}$ according to the last equality in equation (30). The results for $\left|\mathcal{A}_{n}\right|^{2}$ thus obtained are in perfect agreement with those calculated by using the explicit expressions for $\mathcal{A}_{n}$ in terms of generalized Bessel functions [17] (see (42)).

We emphasize the distinct difference in the behavior of even and odd harmonics inside the sphere of "bound state radius" $\kappa^{-1}$. As shown in Figure 2(a), for $r<\kappa^{-1}$ the odd (laserinduced) harmonics decrease with decreasing $r$ and vanish at the origin, whereas the factors $\mathcal{N}_{2 k}(\mathbf{r}) r^{2}$ tend to constant values at $r \rightarrow 0$ (in agreement with the boundary condition (17) for $l=0)$.

\section{Plateau features in the Keldysh approximation for QQES wavefunctions}

\subsection{The KA wavefunctions}

In commonly used derivations, the KA is formulated directly in terms of the ionization amplitude, e.g., by replacement of the exact final (scattering) state in (2) by the Volkov wavefunction. Thus, it is unclear which approximation corresponds to the same level of accuracy as the KA in analyses of other strong-field processes, e.g., such as HHG. For such purposes, it is desirable to derive the KA ionization amplitude in terms of a "KA wavefunction," $\Psi_{\mathrm{KA}}(\mathbf{r}, t)$, which may then be used without any additional approximations in studies of processes other than ionization at the KA level of accuracy. Obviously, the appropriate KA wavefunction should involve information on the initial bound state, $\psi_{0}(\mathbf{r})$, and thus may be obtained as some approximation to an exact solution of the TDSE (1) that evolves from $\psi_{0}(\mathbf{r}, t)$ in a strong laser field. The QQES approach is thus an appropriate one for such a generalization of the KA.

Exact analytical results for $n$-photon ATD amplitudes $\mathcal{A}_{n}$ in the case of short-range potentials (in both the ZRP and TDER models) may be represented $[17,20]$ as a linear combination of generalized Bessel functions multiplied by Fourier coefficients $f_{k}$ of the function $f_{\epsilon}(t)$ (see, e.g., (42)). These amplitudes $\mathcal{A}_{n}$ reduce to the KA amplitudes $\mathcal{A}_{n}^{\mathrm{KA}}$ by neglecting the time- 
dependent function $f_{\epsilon}(t)$ (specifically, by assuming $f_{k}=f_{0} \delta_{k 0}$ ) and by making the substitution $\epsilon \rightarrow E_{0}$. The QQES wavefunction in this approximation follows immediately from (19) :

$$
\begin{aligned}
\Psi_{\mathrm{KA}}(\mathbf{r}, t) & =\Phi_{\mathrm{KA}}(\mathbf{r}, t) \mathrm{e}^{-(\mathrm{i} / \hbar) E_{0} t} \\
& =-\frac{2 \pi \hbar^{2}}{m} f_{0} \lim _{\mathbf{r}^{\prime}=0} \mathcal{Y}_{l m}\left(\nabla_{\mathbf{r}^{\prime}}\right) \int_{-\infty}^{t} G^{V}\left(\mathbf{r}, t ; \mathbf{r}^{\prime}, t^{\prime}\right) \mathrm{e}^{-(\mathrm{i} / \hbar) E_{0} t^{\prime}} \mathrm{d} t^{\prime},
\end{aligned}
$$

where the factor $f_{0}$, which generally is defined by the normalization condition (9), is replaced by $\kappa^{l} C_{\kappa l}$ (cf. (14)). The wavefunction (31) may be called the KA wavefunction in the TDER approach since, indeed, it may be verified that for large $r$ it has the asymptotic form (11) with $\mathcal{A}_{n} \rightarrow \mathcal{A}_{n}^{\mathrm{KA}}$. For a ZRP model, in which case the identity

$$
U(\mathbf{r}) \Phi_{\epsilon}(\mathbf{r}, t)=-\frac{\sqrt{\pi} \hbar^{2}}{m} \delta(\mathbf{r}) f_{\epsilon}(t)
$$

is valid, the definition (31) for $\Psi_{\mathrm{KA}}(\mathbf{r}, t)$ may be rewritten as follows:

$$
\Psi_{\mathrm{KA}}(\mathbf{r}, t)=\Phi_{\mathrm{KA}}(\mathbf{r}, t) \mathrm{e}^{-(\mathrm{i} / \hbar) E_{0} t}=-\frac{\hbar^{2}}{m} \sqrt{2 \pi \kappa} \int_{-\infty}^{t} G^{V}\left(\mathbf{r}, t ; 0, t^{\prime}\right) \mathrm{e}^{-(\mathrm{i} / \hbar) E_{0} t^{\prime}} \mathrm{d} t^{\prime}
$$

or, equivalently,

$$
\Psi_{\mathrm{KA}}(\mathbf{r}, t)=\iint G^{V}\left(\mathbf{r}, t ; \mathbf{r}^{\prime}, t^{\prime}\right) U\left(\mathbf{r}^{\prime}\right) \psi_{0}\left(\mathbf{r}^{\prime}, t^{\prime}\right) \mathrm{d} \mathbf{r}^{\prime} \mathrm{d} t^{\prime} .
$$

This equation may be considered as a general definition of the KA wavefunction as the "zero-order iteration" of the general eigenvalue equation (8) for the QQES wavefunction in which the following two approximations are made on the right-hand side of (8) $: \epsilon \rightarrow E_{0}$ and $\Phi_{\epsilon}\left(\mathbf{r}^{\prime}, t^{\prime}\right) \rightarrow \psi_{0}\left(\mathbf{r}^{\prime}, t^{\prime}\right)$. In terms of the KA wavefunction having the form (33), the SMA result for $S_{\mathrm{KA}}$ in the last formula in equation (3) may be obtained by taking the projection of $\Psi_{\mathrm{KA}}(\mathbf{r}, t)$ on the (final) Volkov state $\Psi_{\mathbf{p}}^{V}(\mathbf{r}, t)$ in the limit $t \rightarrow \infty$. Then, the above-mentioned result for $S_{\mathrm{KA}}$,

$$
S_{\mathrm{KA}}=\lim _{t \rightarrow \infty}\left\langle\Psi_{\mathbf{p}}^{V}(\mathbf{r}, t) \mid \Psi_{\mathrm{KA}}(\mathbf{r}, t)\right\rangle=-\frac{\mathrm{i}}{\hbar} \int_{-\infty}^{\infty}\left\langle\Psi_{\mathbf{p}}^{V}(\mathbf{r}, t)|U(\mathbf{r})| \psi_{0}(\mathbf{r}, t)\right\rangle \mathrm{d} t,
$$

follows immediately using the standard representation for $G^{V}\left(\mathbf{r}, t ; \mathbf{r}^{\prime}, t^{\prime}\right)$ in (33) via the Volkov wavefunctions

$$
G^{V}\left(\mathbf{r}, t ; \mathbf{r}^{\prime}, t^{\prime}\right)=-\frac{\mathrm{i}}{\hbar} \Theta\left(t-t^{\prime}\right) \int \mathrm{d} \mathbf{q} \Psi_{\mathbf{q}}^{V}(\mathbf{r}, t) \Psi_{\mathbf{q}}^{V *}\left(\mathbf{r}^{\prime}, t^{\prime}\right) .
$$

The KA wavefunction (33) for a general potential $U(\mathbf{r})$ may also be represented in an alternative form,

$$
\Psi_{\mathrm{KA}}(\mathbf{r}, t)=\psi_{0}(\mathbf{r}, t)+\int_{-\infty}^{t} \mathrm{~d} t^{\prime} \int \mathrm{d} \mathbf{r}^{\prime} G^{V}\left(\mathbf{r}, t ; \mathbf{r}^{\prime}, t^{\prime}\right) V\left(\mathbf{r}^{\prime}, t^{\prime}\right) \psi_{0}\left(\mathbf{r}^{\prime}, t^{\prime}\right),
$$

using the following formal relations:

$$
G^{V}=G_{0}+G^{V} V G_{0}, \quad G_{0} U \psi_{0}=\psi_{0},
$$

where $G_{0}$ is the (retarded) free-electron Green's function. (Note that the wavefunction (36) was introduced in [29] as an iterative solution of the initial value problem for the TDSE (1) and was employed in HHG calculations.)

Obviously, to obtain an explicit analytical form for $\Psi_{\mathrm{KA}}(\mathbf{r}, t)$ using (33) or (36), the wholespace solution $\psi_{0}\left(\mathbf{r}^{\prime}\right)$ for the potential $U(\mathbf{r})$ is necessary. In the TDER approach, which is independent of the shape of $U(\mathbf{r})$, the KA wavefunction (for the region $r>r_{c}$ ) is given by ex- 
pression (31). Note that the wavefunction (31) is independent of the effective range $r_{l}$ and depends only on the bound state energy $E_{0}$; also, it has the proper spatial symmetry $\left(\sim Y_{l m}(\hat{\mathbf{r}})\right)$ at small $r$, in agreement with (12). Note furthermore that as $F \rightarrow 0$ the large $r$ (asymptotic) form of (31) tends to that for $\psi_{0}(\mathbf{r})=\varphi_{\kappa l m}(r) Y_{l, m}(\hat{\mathbf{r}})$ in (14), while the integral in (32) reduces to the bound state wavefunction $\psi_{0}(\mathbf{r}, t)$ for the ZRP model.

The next-order in $U(\mathbf{r})$ correction to the KA wavefunction may be obtained by straightforward generalization of (33), i.e., by iterating equation (8) and then substituting $\epsilon \rightarrow E_{0}$ and $\Phi\left(\left(\mathrm{r}^{\prime}, \mathrm{t}^{\prime}\right) \rightarrow \psi_{0}\left(\mathbf{r}^{\prime}, t^{\prime}\right)\right.$. In this case, the "IKA wavefunction" is

$$
\begin{aligned}
\Psi_{\text {IKA }}(\mathbf{r}, t)= & \int_{-\infty}^{t} \mathrm{~d} t^{\prime} \int \mathrm{d} \mathbf{r}^{\prime} G^{V}\left(\mathbf{r}, t ; \mathbf{r}^{\prime}, t^{\prime}\right) U\left(\mathbf{r}^{\prime}\right) \int_{-\infty}^{t^{\prime}} \mathrm{d} t^{\prime \prime} \\
& \times \int \mathrm{d} \mathbf{r}^{\prime \prime} G^{V}\left(\mathbf{r}^{\prime}, t^{\prime} ; \mathbf{r}^{\prime \prime}, t^{\prime \prime}\right) U\left(\mathbf{r}^{\prime \prime}\right) \psi_{0}\left(\mathbf{r}^{\prime \prime}, t^{\prime \prime}\right),
\end{aligned}
$$

and its asymptotic form as $r \rightarrow \infty$ yields the $n$-photon ATI amplitude, which follows from the IKA $S$-matrix element (4). In our approach, this $S$-matrix element, in turn, may be obtained similarly to $S_{\mathrm{KA}}$ in (34), substituting $\Psi_{\mathrm{IKA}}(\mathbf{r}, t)$ instead of $\Psi_{\mathrm{KA}}(\mathbf{r}, t)$. Using (37) for $G^{V}\left(\mathbf{r}^{\prime}, t^{\prime}\right.$; $\left.\mathbf{r}^{\prime \prime}, t^{\prime \prime}\right)$ in (38), the IKA wavefunction $\Psi_{\mathrm{IKA}}(\mathbf{r}, t)$ may be written in a form that explicitly involves $\Psi_{\mathrm{KA}}(\mathbf{r}, t)$ and the next-order correction:

$$
\begin{aligned}
\Psi_{\mathrm{IKA}}(\mathbf{r}, t)= & \Psi_{\mathrm{KA}}(\mathbf{r}, t)+\int_{-\infty}^{t} \mathrm{~d} t^{\prime} \int \mathrm{d} \mathbf{r}^{\prime} G^{V}\left(\mathbf{r}, t ; \mathbf{r}^{\prime}, t^{\prime}\right) U\left(\mathbf{r}^{\prime}\right) \int_{-\infty}^{t^{\prime}} \mathrm{d} t^{\prime \prime} \\
& \times \int \mathrm{d} \mathbf{r}^{\prime \prime} G^{V}\left(\mathbf{r}^{\prime}, t^{\prime} ; \mathbf{r}^{\prime \prime}, t^{\prime \prime}\right) V\left(\mathbf{r}^{\prime \prime}, t^{\prime \prime}\right) \psi_{0}\left(\mathbf{r}^{\prime \prime}, t^{\prime \prime}\right) .
\end{aligned}
$$

This equation shows that the $n$-photon ATI amplitude $\mathcal{A}_{n}^{\text {IKA }}$ calculated via the asymptotic form of $\Psi_{\text {IKA }}(\mathbf{r}, t)$ at $r \rightarrow \infty$ (cf.. (11)) involves both the KA amplitude $\mathcal{A}_{n}^{\mathrm{KA}}$ (given by the asymptotic form of $\left.\Psi_{\mathrm{KA}}(\mathbf{r}, t)\right)$ and the high order in $U(\mathbf{r})$ correction to $\mathcal{A}_{n}^{\mathrm{KA}}$ (given by the asymptotic form of the last term on the right-hand side of (39)).

Despite the attractive simplicity of the result (38) for the improved KA wavefunction, we feel that the procedure used for its derivation cannot be considered as a regular method for the "perturbative" account of binding potential effects beyond the KA. The major concern is the use of an iterative approach for a homogeneous (i.e., eigenvalue) equation (8). In view of the absence of an inhomogeneous term (such as the incident wave in the scattering state equation (7)), the approach is definitely not equivalent to the Born expansion in collision problems and it is impossible to formulate any quantitative estimate of its accuracy. Moreover, the eigenvalue, i.e., the complex quasienergy, is approximated by $E_{0}$ and is unchanged in this iterative scheme. Most likely for this reason, the improvement of the KA results for the ATD amplitude by iterative expansion (in the binding potential) of the exact solution of equation (8) for the ZRP model [17] gives results that are generally different from those given by (4) for the same model (although for the low-frequency regime the difference between these two versions of the IKA is not so significant except in the region of the onset of the plateau; see [17] for a more detailed discussion).

As is commonly known, the observables calculated within the strong-field approximation (or the KA), such as ATD and HHG rates, are generally gauge dependent. To clarify this question for the KA in the framework of the TDER approach, we first note that for a ZRP model (i.e., for s-states, $r_{0}=0$ ) the KA ionization amplitudes in both length $(L)$ and velocity $(V)$ gauges are equivalent. This fact was proved a long time ago [39] and is rather evident from the SMA result (3) for the KA amplitude: since the ZRP $U(\mathbf{r})$ reduces the integration over $\mathbf{r}$ in the last term in (3), the gauge-dependent factor, $\exp \left\{(\mathrm{i}|e|) /\left(\hbar \omega^{2}\right) \mathbf{r} \cdot \dot{\mathbf{F}}(t)\right\}$, in the Volkov wavefunction $\Psi_{\mathbf{p}}^{V}(\mathbf{r}, t)$ does not affect the KA amplitude $S_{\mathrm{KA}}$. In the same way, gauge 

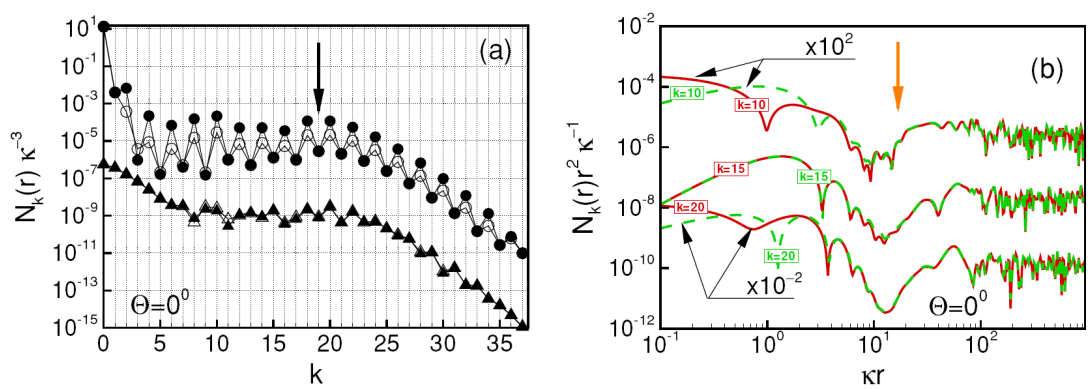

Figure 3. Comparison of exact and KA results for $\mathcal{N}_{k}(\mathbf{r})$ for $\Theta=0^{\circ}$ and the same laser parameters as in Figures 1 and 2. (a) Dependence on $k$ : solid and open circles show exact and KA results for $r=0.1 \kappa^{-1}$; solid and open triangles show exact and KA results for $r=5 \kappa^{-1}$. (b) Dependence on $r$ : solid lines show exact results; dashed lines show KA results. Results for $k=$ 10 and 20 are multiplied by factors of $10^{2}$ and $10^{-2}$, respectively. Arrows show the positions of $k_{\max }=\left(\left|E_{0}\right|+3.17 u_{p}\right) /(\hbar \omega)$ in (a), and $r=\alpha_{0}=(|e| F) /\left(m \omega^{2}\right)$ in (b).

invariance also holds for the improved KA amplitude (4) for a ZRP model. For p-states, as was noted in [20], only the $L$-gauge KA wavefunction (31) matches the exact TDER wavefunction $\Psi_{\epsilon}(\mathbf{r}, t)$. The qualitatively different $V$-gauge KA predictions for the low-energy (or "Keldysh") part of ATD spectra from p-states as compared to those calculated in the $L$-gauge KA and to exact results have been demonstrated recently in [22] (for the TDER model) and in [40] (by comparison of results obtained by numerical solution of the TDSE with those in the "ZRP-like" model for p-states, i.e., using the asymptotic form (14) for $l=1$ as the wavefunction of a p-state in the whole space, $0<r<\infty$ ).

\subsection{Plateau features in $K A$ wavefunctions}

In Figure 3, we present a comparison between two sets of $\mathcal{N}_{k}(\mathbf{r})$ (cf. (23) and (24)): one calculated using the exact QQES wavefunction, as in Figures 1(a) and 2(a), and the other using the KA wavefunction $\Phi_{\mathrm{KA}}(\mathbf{r}, t)$ in (32). The most surprising feature of the data in Figure 3 is that plateau structures in the spectra of Fourier (or QES) harmonics, $\Phi_{n}(\mathbf{r})$, exist even in the $\mathrm{KA}$, i.e., in the QES harmonics $\Phi_{n}^{\mathrm{KA}}(\mathbf{r})$ of $\Phi_{\mathrm{KA}}(\mathbf{r}, t)$. Moreover, surprisingly excellent quantitative agreement is observed between the exactly calculated factors $\mathcal{N}_{k}(\mathbf{r})$ for the case of odd $k(k=2 s+1)$ and those calculated in the KA, for both their $k$ - and $r$-dependences. For even $k$, similarly good agreement is observed only for distances far beyond the radius of the bound state, $\kappa^{-1}$. The explanation for this qualitative difference in the behavior of the exact and KA results for even $k$ at $r \leq \kappa^{-1}$ in Figure 3(b) is the same as for the difference between the exact even and odd QES harmonics in Figure 2(a), i.e., in the KA, both even (with the exception of $k=0)$ and odd QES harmonics $\Phi_{k}^{\mathrm{KA}}(\mathbf{r})$ are laser-field induced, so that the KA results in Figure 3(b) for both odd $(k=15)$ and even $k(k=10$ and $k=20)$ have similar (decreasing) behavior with decreasing $r$.

This comparison of exact and KA results allows one to make some conclusions on the key features of a QQES wavefunction $\Psi_{\epsilon}(\mathbf{r}, t)$ describing the quasistationary state that develops from an initial, weakly bound state $\psi_{0}(\mathbf{r})=\varphi_{\kappa l m}(r) Y_{l m}(\hat{\mathbf{r}})$ under the influence of a strong monochromatic field. An accurate numerical analysis within the TDER approach shows that only a finite number of QES harmonics $\Phi_{s}(\mathbf{r})$ contribute significantly to the Fourier expansion (5) of $\Psi_{\epsilon}(\mathbf{r}, t)$ in the strong-field limit. These are the harmonics with $s$ from 0 up to $s=$ $s_{\max }$, where $s_{\max } \approx\left(\left|E_{0}\right|+3.17 u_{p}\right) /(\hbar \omega)$. Odd $(s=2 k)$ and even $(s=2 k+1)$ harmonics have op- 
posite spatial parity (see (22)) and essentially different behavior in the region of the localized bound state, i.e., $r \leq \kappa^{-1}$ : the odd harmonics decrease with decreasing $r$, while the even ones increase. For $r>\kappa^{-1}$, all harmonics with $s \leq s_{\max }$ have a similar, regular behavior up to $\mathrm{r}$ $\approx \alpha_{0}=(|e| F) /\left(m \omega^{2}\right)$, beyond which the behavior is irregular and highly oscillatory. The exact TDER wavefunction (19) may also be represented as a linear combination of $2 \hbar \omega$-spaced (in energy) KA-like constituents multiplied by $f_{s} \equiv f_{2 k}$ :

$$
\Psi_{\epsilon}(\mathbf{r}, t)=\mathrm{e}^{-(\mathrm{i} / \hbar) \epsilon t} \Phi_{\epsilon}(\mathbf{r}, t)=\sum_{s} f_{s} \Psi_{\epsilon+s \hbar \omega}^{\mathrm{KA}}(\mathbf{r}, t),
$$

where $\Psi_{\epsilon}^{\mathrm{KA}}{ }_{\epsilon}(\mathbf{r}, t)$ is defined by direct comparison with the right-hand side of (19). (Since $\Psi_{\epsilon+s \hbar \omega}^{\mathrm{KA}}(\mathbf{r}, t)$ differs from $\Psi_{\mathrm{KA}}(\mathbf{r}, t)$ (cf. (31)) only by the energy parameter $\epsilon+s \hbar \omega$, plateau structures in its QES harmonics are similar to those for $\Phi_{n}^{\mathrm{KA}}(\mathbf{r})$, except that the extent of the plateau region in $n$ (given by $n_{\max } \approx\left(\left|E_{0}\right|+3.17 u_{p}\right) /(\hbar \omega)$ for $\Phi_{n}^{\mathrm{KA}}(\mathbf{r})$ ) becomes shorter with increasing $s$, i.e., $n_{\max } \rightarrow\left(n_{\max }-s\right)$.) The (even) coefficients $f_{s}$ originate from binding potential effects and have plateau-like behavior up to the same cut-off value, $s_{\max }$, as that for $\Phi_{s}(\mathbf{r})$. The simplest approximation to $\Psi_{\epsilon}(\mathbf{r}, t)$ provides the KA wavefunction $\Psi_{\mathrm{KA}}(\mathbf{r}, t)(31$; see also $33)$, i.e., the term with $s=0$ in (40). Although this approximation corresponds to the minimal account of binding potential effects, QES harmonics of $\Phi_{\mathrm{KA}}(\mathbf{r}, t)$ perfectly reproduce the exact harmonics $\Phi_{s}(\mathbf{r})$ for odd $s$, while for even $s$ they fail to describe the behavior of $\Phi_{s}(\mathbf{r})$ at small $r, r<\kappa^{-1}$. Thus, the behavior of the even harmonics $\Phi_{2 k}(\mathbf{r})$ is governed by the bindingpotential-mediated coefficients $f_{2 k}$ (or by rescattering effects, in terms of the RS).

\section{Relation between plateaus in QQES harmonics and those in ATD and HHG spectra}

\subsection{ATD}

From the representation (40) for the QQES wavefunction and its asymptotic form (11), it follows that the exact result for the $n$-photon ATD amplitude may be expressed as a sum of partial, "KA-like" amplitudes $A_{n-2 k}^{\mathrm{KA}}$ :

$$
\mathcal{A}_{n}=\sum_{k=-\infty}^{\infty} f_{2 k} A_{n-2 k}^{\mathrm{KA}},
$$

where the KA amplitude, $\mathcal{A}_{n}^{\mathrm{KA}}$, is given by the term with $s=0: \mathcal{A}_{n}^{\mathrm{KA}} \equiv f_{0} A_{n}^{\mathrm{KA}}\left(\epsilon=E_{0}\right)$. An explicit form for $A_{p}^{\mathrm{KA}}$ in (41) in terms of generalized Bessel functions $\mathcal{J}_{k}(x, y)$ may be found in [17] (for s-states) and in [20] (for p-states). For example, for ATD from an s-state $\psi_{0}(\mathbf{r})$ we have [17]

$$
\mathcal{A}_{n}=\mathrm{i}^{n} \sum_{k=-\infty}^{\infty}(-1)^{k} f_{2 k} \mathcal{J}_{n-2 k}\left(\frac{|e| F k_{n}}{m \omega^{2}}(\hat{\epsilon} \cdot \mathbf{n}), \frac{u_{p}}{2 \hbar \omega}\right),
$$

where $\hbar k_{n}=\left(2 m\left(\epsilon+n \hbar \omega-u_{p}\right)\right)^{1 / 2}, \mathbf{n}$ is the electron ejection direction, and $\mathcal{J}_{n-2 k}(x, y)$ is given by

$$
\mathcal{J}_{v}(x, y)=\sum_{p=-\infty}^{\infty} J_{2 p+v}(x) J_{p}(y) .
$$

In general, the sum over $k$ in (41), (42) runs from $-\infty$ to $\infty$. However, the actual number of coefficients $f_{2 k}$ that contribute significantly depends on the regime of ionization. In Figure 4 , these regimes are illustrated for ATD of $\mathrm{F}^{-}$for four intensities. In the weak-field (perturbative) regime, the behavior of the coefficients $f_{s}$ predicted by perturbation theory (PT) is $f_{s} \approx$ 

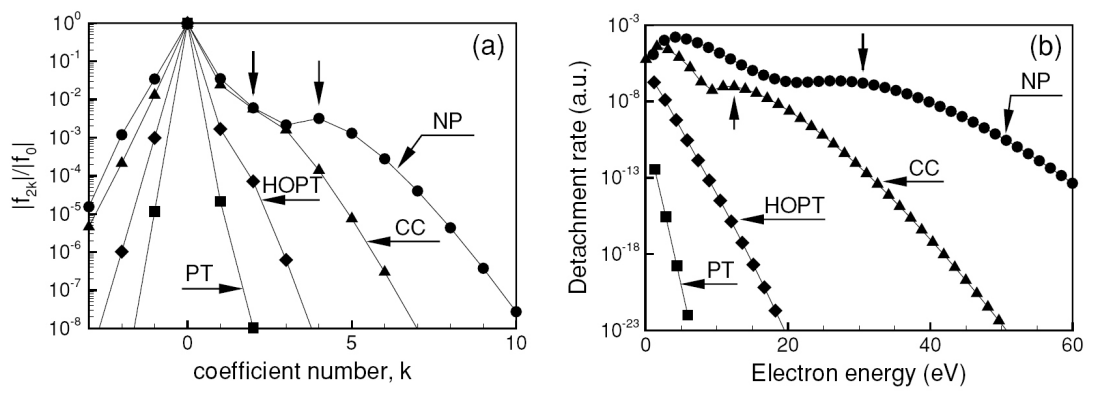

Figure 4. ATD spectra for $\mathrm{F}^{-}$for $\lambda=800 \mathrm{~nm}\left(\hbar \omega=0.46\left|E_{0}\right|\right)$ and four intensities. (a) Spectrum of $f_{n}(n=2 k)$ coefficients (cf. (41)) and (b) spectrum of ATD detachment rates (for electrons ejected along the laser polarization axis). Squares (PT) $: I=1.85 \times 10^{10} \mathrm{~W} \mathrm{~cm}^{-2}$; diamonds $\left(\right.$ HOPT) $: I=1.56 \times 10^{12} \mathrm{~W} \mathrm{~cm}^{-2}$; triangles $(\mathrm{CC}): I=1.93 \times 10^{13} \mathrm{~W} \mathrm{~cm}^{-2}$; circles $(\mathrm{NP}): I=5.2 \times 10^{13} \mathrm{~W} \mathrm{~cm}^{-2}$. Arrows mark the "classical cut-offs": $(\mathrm{a})\left(\left|E_{0}\right|+3.17 u_{p}\right) /(\hbar \omega)$ and (b) $10 u_{p}$

$\alpha s(\omega) \mathrm{F}^{|s|}$, i.e., $f_{s}$ decreases rapidly with increasing $|s|$. The $n$-photon ATD rates $\Gamma_{n}$ decrease similarly: $\Gamma_{n} \approx \beta n(\omega) I^{n}$ (cf. the PT curves in Figures 4(a) and 4(b)). In this regime, the PT expansions of the terms $A_{n-2 k}^{\mathrm{KA}}$ as well as of the coefficients $f_{2 k}$ (for both positive and negative $k$ ) in (41) must be taken into account in order to calculate the factor $\beta_{n}(\omega)$ correctly. Use of only the PT expansion for the KA amplitude, $\mathcal{A}_{n}^{\mathrm{KA}}$, is not enough for this purpose. In other words, the magnitude of $\mathcal{A}_{n}$ in the PT regime is essentially determined by binding potential effects, as might be expected. With increasing intensity, high-order PT (HOPT) corrections become important; in this regime, a larger number of terms in the PT expansions of $f_{2 k}$ and $A_{n-2 k}^{\mathrm{KA}}$ must be taken into account and their contributions change the slope of the HOPT curves compared to the PT curves in Figure 4. The first signature of plateau effects appears at the intensity for which $u_{p} \approx \hbar \omega$ (i.e., when the lowest open ionization channel is closed and thus the PT expansions become divergent [35]). This regime is marked by the "channel closing" (CC) curves in Figure 4. Finally, in the strongly non-perturbative (NP) regime, $u_{p}>\hbar \omega$, only a limited number of coefficients $f_{s}$ (i.e., those in the plateau-like region with $0 \leq s \leq s_{\max }$ in Figure 4(a)) contribute significantly. Correspondingly, the ATD rates $\Gamma_{n}$ have a well-developed plateau structure (cf. the NP curves in Figures 4(a) and 4(b)) that originates entirely from that in the spectrum of $f_{2 k}$ coefficients.

In principle, the general expression (30) for $\Gamma_{n}$ in terms of the coefficients $A_{k, n}$ in the asymptotic form (28) of the QES harmonics $\Phi_{n}(\mathbf{r})$ may also be used to calculate ATD rates. This representation may be more convenient than (41) for obtaining PT expansions of $\Gamma_{n}$ in the weak-field limit. However, in the strong-field regime, the representation (41) is preferable, since in this regime the exact amplitude $\mathcal{A}_{n}$ is well approximated by a coherent superposition of only a finite number of binding-potential-induced KA terms,

$$
\mathcal{A}_{n}=\sum_{s=0}^{s_{\max }} f_{s} A_{n-s}^{\mathrm{KA}}\left(\epsilon=E_{0}\right),
$$

where $s$ takes only even values, $s=2 k$. This result confirms the interpretation of $\mathcal{A}_{n}$ as a coherent superposition of the $n$-photon ionization amplitude from the ground state $(s=0)$ and the $(n-2 k)$-photon ionization amplitudes from the KA harmonics $\underset{\epsilon+2 k \hbar \omega}{\mathrm{KA}}(\mathbf{r}, t)$ in (40), whose "population amplitudes" are given by the coefficients $f_{s=2 k}$. The contribution of the KA amplitude (i.e., the $s=0$ term) to (44) decreases rapidly (exponentially in the tunneling regime) with increasing detached electron energy, so that the terms with $s>0$ in (44) become 
dominant for higher electron energies (despite the fact that $\left.\left|f_{s}\right| \ll\left|f_{0}\right|\right)$. Moreover, the interference of the $s=0$ term with the others is significant only at the onset of the high-energy plateau, whereas the high-energy part of the plateau originates entirely from terms with $0 \ll s \leq s_{\max }$.

In the quantum (QQES) language, equations (40) and (44) support the following "twostep," quantum scenario for ATD in the strong-field regime: the first step, which follows from general arguments of QES theory [34], occurs within a few cycles after the turn on of a strong monochromatic pulse. Namely, the quasienergy structure of the QQES wavefunction in (5) is established by means of fast (nonadiabatic) multiphoton absorption or stimulated emission of photons by an initially bound electron. In the TDER model, this wavefunction may be expressed in the remarkable form (40), partitioning the infinite sum of QES harmonics $\Phi_{s}(\mathbf{r})$ in (5) in terms of KA harmonics $\Psi_{\epsilon+s \hbar \omega}^{\mathrm{KA}}(\mathbf{r}, t)$. The origin of these KA harmonics is different for zero and non-zero $s$. For $s=0$, the KA harmonic is generated by the laser field from the initial bound state $\psi_{0}(\mathbf{r}, t)$ neglecting binding potential effects (it is the term with $s=0$ in (40) or the KA wavefunction). For $s>0$, the (generally infinite) set of $2 \hbar \omega$-spaced KA harmonics is generated by the binding-potential-induced, multiphoton absorption of even numbers $(s=$ $2 k$ ) of photons by the initially bound electron near the origin. Owing to the plateau structure in the spectrum of $f_{s}$, only a finite number of KA harmonics (which, in terms of the RS, may be called "rescattering harmonics") becomes highly populated in the strong-field limit (i.e., those with $0 \leq s \leq s_{\max }$ ). Thus, in the strong-field regime, the first step of the quantum scenario for ATD consists in the generation of the set of almost equally populated KA harmonics $\Psi_{\epsilon+\operatorname{s} \hbar \omega}^{\mathrm{KA}}(\mathbf{r}, t)$ with numbers $s$ up to $s_{\max } \approx\left(\left|E_{0}\right|+3.17 u_{p}\right) /(\hbar \omega)$. In the second step, the coherent superposition of these highly populated KA harmonics decays slowly in the laser field (as compared to the laser period $T=(2 \pi) / \omega$ ) with amplitudes $A_{n-s}^{\mathrm{KA}}$ (cf.. (44)), e.g., by means of tunneling or barrier-suppressed ionization. Although the tunneling concept is generally applicable only in the low-frequency regime, $\hbar \omega \ll\left|E_{0}\right|$, an analysis in [22] shows that typical plateau features in ATD spectra also exist in the non-tunneling (multiphoton) regime of high frequencies, $\hbar \omega \leq\left|E_{0}\right|$.

Note, finally, that the cut-off position of plateau structures in all cases is intimately related to the famous $3.17 u_{p}$-law, which has a purely classical origin since $\mathcal{E}_{\mathrm{cl}}=(3.17 \ldots) u_{p}$ is the maximum energy gained by a free electron moving along a closed classical trajectory in a laser field. (Regarding the connection between the $\sim 10 u_{p}$-law for the cut-off position in ATD spectra and the $3.17 u_{p}$-rule, see [19].) The appearance of this manifestly classical quantity in our completely quantum analysis is not surprising since it may be expected that with increasing intensity of the laser field (treated classically) some classical features in the strongfield limit should appear even in a rigorous quantum analysis. Specifically, in the TDER approach, the quantity $\mathcal{E}_{\mathrm{cl}}$ is "hidden" in the classical action $S$ that enters both the equations for the QQES wavefunction (cf.. (19) and (21)) and the function $f_{\epsilon}(t)$ (cf. (16)). In particular, the action $S(t, t-\tau) \equiv S\left(\mathbf{r}=0, t ; \mathbf{r}^{\prime}=0, t-\tau\right)$ involves all closed classical trajectories starting from the origin at the moment $t-\tau$ and having a return time $\tau$. Thus, the integral in (16) may be interpreted as an integral over all return times. $\mathcal{E}_{\mathrm{cl}}$ may be deduced by maximizing the energy gain, $\Delta \mathrm{E}=-\partial S(0, t ; 0, t-\tau) / \partial t$, over the set of return times $\tau$. It corresponds to the shortest return time $\tau=\tau_{0}\left(\omega \tau_{0}=4.086 \ldots\right)$.

\section{2. $H H G$}

The quantum interpretation of plateau features in HHG spectra is quite different from that for ATD. On the one hand, all previous SMA calculations of HHG spectra have evaluated the induced dipole moment and HHG rates using a field-dressed bound state wavefunction on the 
level of the KA. For example, the KA wavefunction (36) has been used to calculate HHG rates in [29] and the results are in good quantitative agreement with those calculated in [41], which employ another form (32) of the KA wavefunction. On the other hand, exact QQES calculations of HHG rates based on equations (10) within the ZRP model [42] predict HHG plateaus that are in excellent agreement with those obtained in the KA. Thus arises a puzzling question which, as far as we know, has not yet been discussed: why are plateau features in HHG described so well within the KA, while high-order binding potential effects must be included in order to describe plateaus in ATI spectra? The answer to this question may be found by considering the structure of the exact and KA wavefunctions described in sections 4 and 5 .

As discussed in section 2.2, the HHG rates, $\mathcal{R}_{N}$, in (10) are defined by the corresponding Fourier components, $\tilde{\mathbf{d}}_{N}$, of the dual dipole moment $\tilde{\mathbf{d}}(t)$

$$
\tilde{\mathbf{d}}(t)=\left\langle\tilde{\Phi}_{\epsilon}(\mathbf{r}, t)|\mathrm{d}| \Phi_{\epsilon}(\mathbf{r}, t)\right\rangle .
$$

In terms of the QES harmonics $\Phi_{s}(\mathbf{r})$ of $\Phi_{\epsilon}(\mathbf{r}, t)$, the result for $\tilde{\mathbf{d}}_{N}$,

$$
\tilde{\mathbf{d}}_{N}=\sum_{s=-\infty}^{\infty}\left\langle\tilde{\Phi}_{s-N}|\mathbf{d}| \Phi_{s}\right\rangle=\int \mathbf{d} \mathcal{N}_{N}(\mathbf{r}) \mathrm{d} \mathbf{r},
$$

involves an infinite summation over bilinear combinations of QES harmonics having opposite spatial parities, $(-1)^{s}$ and $(-1)^{s+N}$, in accordance with (22) for the case of an s-state, where $N=2 k+1$. (Note that according to the definition of the dual function below equation (9), we have $\tilde{\Phi}_{s-N}^{\square}(\mathbf{r})=\Phi_{s-N}(\mathbf{r})$ for s-states.)

The number of terms contributing to the sum over $s$ in the exact result (46) for $\tilde{\mathbf{d}}_{N}$ depends on the laser intensity (similarly to that for the sum over $k$ in the exact result (41) for the ATD amplitude). In the perturbative regime, all (even and odd) QES harmonics with $|s| \leq$ $N$ contribute on an equal footing to the lowest order PT result for the nonlinear susceptibility $\chi_{N}(N \omega), \tilde{\mathbf{d}}_{N}=\mathbf{e}_{z} \chi_{N}(N \omega) F^{N}$. Taking into account higher order PT corrections $\chi_{N+2 k}(N \omega) \sim$ $\left.I^{k}\right)$ to $\chi_{N}(N \omega)$, the number of contributing terms in the sum over $s$ in (46) increases. The loworder harmonics generated in these regimes exhibit a typically perturbative spectrum (see the curves PT and HOPT in Figure 5(b)). The use of the KA wavefunction instead of the exact one for calculations of $\tilde{\mathbf{d}}(t)$ leads to significantly different results for the frequency dependence of $\chi_{N}(N \omega)$. For example, consider the lowest order PT results for $N=3$, in which case $\mathbf{d}_{3}$ has the following general form (in au) :

$$
\mathbf{d}_{3}=\mathbf{e}_{z} \chi_{3}(3 \omega) F^{3}, \quad \chi_{3}(3 \omega)=-\frac{\kappa^{6}}{2160 \tilde{\omega}^{8}}[\alpha(\tilde{\omega})+\alpha(-\tilde{\omega})], \quad \tilde{\omega}=\frac{\hbar \omega}{\left|E_{0}\right|} .
$$

The exact result for the function $\alpha(\tilde{\omega})$ is [43]

$$
\begin{gathered}
\alpha(\tilde{\omega})=1-3(1+\tilde{\omega})^{5 / 2}+3(1+2 \tilde{\omega})^{5 / 2}-(1+3 \tilde{\omega})^{5 / 2}-\frac{5}{6 \tilde{\omega}}[1+\sqrt{1+2 \tilde{\omega}}]\left[1-2(1+\tilde{\omega})^{3 / 2}\right. \\
\left.+(1+2 \tilde{\omega})^{3 / 2}\right]\left[1-(1-\tilde{\omega})^{3 / 2}+(1+2 \tilde{\omega})^{3 / 2}-(1+3 \tilde{\omega})^{3 / 2}\right]
\end{gathered}
$$

while in the KA the function $\alpha(\tilde{\omega})$ has a much simpler analytical structure,

$$
\begin{aligned}
\alpha_{\mathrm{KA}}(\tilde{\omega})=1- & 3(1+\tilde{\omega})^{5 / 2}+3(1+2 \tilde{\omega})^{5 / 2}-(1+3 \tilde{\omega})^{5 / 2}+\frac{45 \tilde{\omega}^{2}}{16}[1-\sqrt{1+2 \tilde{\omega}}] \\
+ & \frac{5 \tilde{\omega}}{4}\left[-7(1+\tilde{\omega})^{3 / 2}+2(1+2 \tilde{\omega})^{3 / 2}+(1+3 \tilde{\omega})^{3 / 2}\right] .
\end{aligned}
$$

Though "on average" the difference between $\chi_{3}^{\mathrm{KA}}(3 \omega)$ and $\chi_{3}(3 \omega)$ is not so large, near the even ionization channel, $2 \hbar \omega \approx\left|E_{0}\right|, \chi_{3}^{\mathrm{KA}}(3 \omega)$ is less than $\chi_{3}(3 \omega)$ by more than an order of 

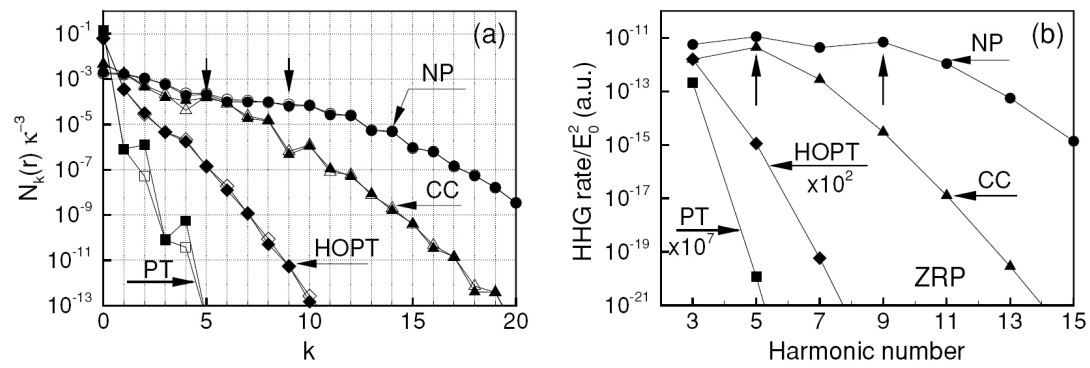

Figure 5. Comparison of (a) the $k$-dependence of the electron density factors $\mathcal{N}_{k}(\mathbf{r})$ (for parallel geometry, $\Theta=0^{\circ}$ and $r=0.5 \alpha_{0}$ ); and (b) the HHG spectrum for the ZRP model, $\hbar \omega=$ $0.46\left|E_{0}\right|$. Squares $(\mathrm{PT}): F=0.01 F_{0}$; diamonds $(\mathrm{HOPT}): F=0.1 F_{0}$; triangles $(\mathrm{CC}): F=0.4 F_{0}$; circles (NP): $F=0.65 F_{0}$, where $F_{0}=\left(2 m\left|E_{0}\right|^{3}\right)^{1 / 2} /(|e| \hbar)$. Arrows mark the "classical cut-offs," $k_{\max }=N_{\max } \approx\left(\left|E_{0}\right|+3.17 u_{p}\right) /(\hbar \omega)$. Open symbols in (a): KA results. Note that $r=0.5 \alpha_{0}$ is equivalent to $r=0.047 \kappa^{-1}$ for $F=0.01 F_{0}, r=0.47 \kappa^{-1}$ for $F=0.1 F_{0}, r=1.89 \kappa^{-1}$ for $F=0.4 F_{0}$, and $r=3.07 \kappa^{-1}$ for $F=0.65 F_{0}$.

magnitude [42]. This result is qualitatively clear since the even QES harmonics of the KA wavefunction are incorrect for $r \leq \kappa^{-1}$.

The number of significant terms that contribute to the sum over $s$ in (46) changes significantly when the intensity increases to values such that $u_{p} \geq \hbar \omega$, at which the lowest multiphoton ATI/ATD channels become closed. The first signature of plateau features in the HHG spectrum appears at $u_{p} \approx \hbar \omega$ (curve CC in Figure 5(b)), and in this (CC) regime the contributions of the QES harmonics $\Phi_{k}(\mathbf{r})$ having positive $k$ become dominant in the sum over $s$ in (46). With further increases in I (i.e., in the strong-field regime; cf. the curve NP in Figure $5(\mathrm{~b})$ ), the single (odd) QES harmonic $\Phi_{N}(\mathbf{r})$ of $\Phi_{\epsilon}(\mathbf{r}, t)$ (i.e., the term with $s=N$ in (46)) gives the dominant contribution to $\tilde{\mathbf{d}}_{N}$. In other words, the amplitude of the $N$ th harmonic, calculated as

$$
\tilde{\mathbf{d}}_{N}=\left\langle\tilde{\Phi}_{0}|\mathbf{d}| \Phi_{N}\right\rangle \approx\left\langle\psi_{0}|\mathbf{d}| \Phi_{N}\right\rangle=\frac{1}{T} \int_{0}^{T}\left\langle\psi_{0}(\mathbf{r})|\mathbf{d}| \Phi_{\epsilon}(\mathbf{r}, t)\right\rangle \mathrm{e}^{\mathrm{i} N \omega t} \mathrm{~d} t
$$

gives an excellent approximation to the exact result in the strong-field regime. Moreover, a similarly good approximation is obtained by the substitution $\Phi_{\epsilon}(\mathbf{r}, t) \rightarrow \Phi_{\mathrm{KA}}(\mathbf{r}, t)$ in (50), i.e., approximating $\tilde{\mathbf{d}}_{N}$ by

$$
\mathbf{d}_{N}^{\mathrm{KA}}=\frac{1}{T} \int_{0}^{T}\left\langle\psi_{0}(\mathbf{r})|\mathbf{d}| \Phi_{\mathrm{KA}}(\mathbf{r}, t)\right\rangle \mathrm{e}^{\mathrm{i} N \omega t} \mathrm{~d} t .
$$

The results obtained within this approximation are in excellent agreement with those in Figure 5(b). (The applicability of the approximate result (51) for analyses of HHG spectra in the strong-field limit was also confirmed by quasiclassical calculations [29].) The explanation of this coincidence is obvious, since the odd QES harmonics of $\Phi_{\mathrm{KA}}(\mathbf{r}, t)$ (which are the only ones that contribute to (51)) nicely agree with the exact QES harmonics over the whole region, $0<r<\infty$. Comparison in Figure 5(a) of exact and KA results for $\mathcal{N}_{k}(\mathbf{r})$ at $r=0.5 \alpha_{0}$ for the same four intensities as in Figure 5(b) shows that the difference is considerable only for even $k$ in the PT regime. The evolution of HHG spectra with increasing intensity, as shown in Figure 5(b), is qualitatively similar to that for the ATD spectra in Figure 4(b); note that the data in both figures are for the same (non-tunneling) laser frequency, $\hbar \omega=0.46\left|E_{0}\right|$. 
In terms of radial matrix elements for HHG and ATI amplitudes, the explanation of the distinct difference in the sensitivity of these amplitudes to the account of binding potential effects may be as follows. Similarly to the case of matrix elements for bound-bound dipole transitions, one may expect that the dominant contribution to HHG matrix elements comes from the region of $r$ up to $r \approx \alpha_{0}$. For such $r$, the QES harmonics $\Phi_{s}^{\mathrm{KA}}(\mathbf{r})$ of $\Phi_{\mathrm{KA}}(\mathbf{r}, t)$ have a well-developed plateau structure in $s$ (up to $\mathrm{s} \approx s_{\max }$ ) and the electron density factors $\mathcal{N}_{k}(\mathbf{r})$ have comparable magnitudes (cf. Figure 2(a)). Thus, the plateau features in HHG spectra are a reflection of those in the spectrum of $\Phi_{s}^{\mathrm{KA}}(\mathbf{r})$, whereas the higher KA constituents of the exact wavefunction (40) (having plateau features similar to those in $\Phi_{\mathrm{KA}}(\mathbf{r}, t)$ ) have little consequence since the coefficients $f_{s}$ in the plateau region are a few orders of magnitude smaller than $f_{0}$. As for the radial matrix elements for the ATD amplitudes, the magnitude of these matrix elements is sensitive to $r$ at small distances (i.e., within the sphere of radius $r \approx \kappa^{-1}$ ). Moreover, this sensitivity increases with increasing electron energy in view of the increased oscillations of the continuum wavefunctions (which is similar to the behavior of radial matrix elements for the ordinary photoeffect). Since plateau structures in the KA wavefunction vanish at small $r$, these wavefunctions fail to reproduce the high-energy plateau in ATD spectra.

\section{Discussion and conclusions}

The action of a long monochromatic pulse on an electron in a bound state $\psi_{0}(\mathbf{r})$ of energy $E_{0}$ results in the generation of an infinite "frequency comb" of QES harmonics $\Phi_{s}(\mathbf{r})$ corresponding roughly to the "energies" $E_{0}+s \hbar \omega$ (approximating $\epsilon$ by $E_{0}$ ); i.e., $\psi_{0}(\mathbf{r}, t)$ evolves to $\Phi_{\epsilon}(\mathbf{r}, t)$ in (5). This "steady" (oscillatory) state is established after the smooth (adiabatic) turn on of the monochromatic field. Its wavefunction $\Phi_{\epsilon}(\mathbf{r}, t)$ may be used to describe any nonlinear phenomenon involving the interaction of an initially bound electron with a laser field. In this paper, we have analyzed an exactly solvable quantum model describing a weakly bound electron in a strong monochromatic laser pulse. Analytical results for the QQES wavefunction $\Phi_{\epsilon}(\mathbf{r}, t)$ in this model have been employed to provide an accurate quantum analysis of plateau features in both HHG and ATI/ATD. Our results show that these characteristic strongfield features originate from similar, plateau-like features in the $s$ - and $\mathbf{r}$-dependences of Fourier (or QES) harmonics, $\Phi_{s}(\mathbf{r})$, of the wavefunction $\Phi_{\epsilon}(\mathbf{r}, t)$ itself.

Specifically, in the strong-field limit $\left(u_{p} \gg \hbar \omega\right)$, the electron density in QES harmonics $\Phi_{s}(\mathbf{r})$ has a typical plateau-like behavior over a wide interval of $r, r \leq \alpha_{0}\left(\alpha_{0}=(|e| F) /\left(m \omega^{2}\right)\right)$ and also over a wide interval of harmonic numbers, $0 \leq s \leq s_{\max }$, with cut-off value $s_{\max } \approx$ $\left(\left|E_{0}\right|+3.17 u_{p}\right) /(\hbar \omega)$. We have already discussed in section 6.1 the classical origin of this latter cut-off value; however, it is worthwhile here to emphasize that it is "laser-field induced," i.e., it is not related to a potential $U(\mathbf{r})$ and originates from the motion of an electron along closed classical trajectories in a laser field. Similarly, outside the region of $U(\mathbf{r})$ (i.e., for $\mathrm{r} \gg$ $r_{c}$ ), plateau features in $\Phi_{s}(\mathbf{r})$ are also laser-field induced since they are perfectly reproduced using the (approximate) KA wavefunction, $\Phi_{\mathrm{KA}}(\mathbf{r}, t)$, instead of $\Phi_{\epsilon}(\mathbf{r}, t)$. This KA wavefunction corresponds to a "minimal" account of the potential, $U(\mathbf{r})$, in that it assumes the initial state is described by the bound state wavefunction, $\psi_{0}(\mathbf{r}, t)$, of the potential $U(\mathbf{r})$. Moreover, odd QES harmonics of $\Phi_{\mathrm{KA}}(\mathbf{r}, t)$ also coincide with $\Phi_{s=2 k+1}(\mathbf{r})$ for $0<r<\kappa^{-1}$, and they both vanish at the origin, $r \rightarrow 0$. Thus, the entire difference between the exact and KA wavefunctions (and thus the influence of high-order binding potential effects) stems only from the behavior of their even QES harmonics near (and at) the origin, $0 \leq r \leq \kappa^{-1}$. In the KA, these even harmonics disappear for $r \rightarrow 0$ (as is the case for the odd harmonics), while the magnitude of $\Phi_{s=2 k}(\mathbf{r})$ for $r \rightarrow 0$ is proportional to the Fourier coefficients $f_{s=2 k}$. These coefficients 
originate from binding potential effects beyond the KA and they also have a plateau structure in the interval $0<s \leq s_{\max }$. Therefore, in terms of the wavefunction, a proper account of the potential $U(\mathbf{r})$ causes the generation of even QES harmonics of the exact QQES wavefunction (spaced in energy by $2 \hbar \omega$ ) near the origin, i.e., exactly in the region where the potential $U(\mathbf{r})$ dominates over $V(\mathbf{r}, t)$. It is significant that away from the origin these "potential-induced" monochromatic harmonics of frequency s $\omega$ become subjected to "field dressing," i.e., they develop into the "KA harmonics," which have exactly the same temporal and spatial dependence as the KA wavefunction, but only a shifted energy, $E_{0}+s \hbar \omega$ (cf. 19). In terms of the RS, these KA harmonics may be called "rescattering harmonics." Thus, in the strongfield limit, the exact QQES wavefunction may be presented in the attractive form (40), in which $s$ runs from 0 to $s_{\max }$, i.e., as a "comb" of rescattering harmonics with energies $E_{s} \equiv E_{0}$ $+s \hbar \omega$ up to $E_{0}+s_{\max } \hbar \omega$. Moreover, most of these energies lie in the continuum, $E_{s}>0$ (i.e., those with $\left.s>s_{\min } \approx\left|E_{0}\right| /(\hbar \omega)\right)$ and, remarkably, the coefficients $f_{s}$ with $s_{\min } \leq s \leq s_{\max }$ (i.e., the "populations" of these KA harmonics in (40)) have a plateau-like structure.

The general properties of the QQES wavefunction $\Phi_{\epsilon}(\mathbf{r}, t)$ discussed above allow one to present a transparent quantum interpretation of plateaus in ATD and HHG spectra. Plateau structures in ATD spectra originate from those in the spectrum of even QES harmonics $\Phi_{2 k}(\mathbf{r})$ near the origin (i.e., in the spectrum of coefficients $f_{2 k}$ ). The population of even QES harmonics in this region is caused exclusively by the binding-potential-induced, multiphoton absorption of an even number of photons. Thus, the quantum treatment of ATD plateau features is consistent with the RS, in which the increase of electron energy along the plateau occurs by means of rescattering events, which take place near the origin and are influenced by the potential $U(\mathbf{r})$.

The situation regarding the origin of the plateau in HHG spectra is more intriguing. The dipole moment component for the harmonic frequency $N \omega$ (where $N$ is an odd integer) involves only odd QES harmonics $\Phi_{s}(\mathbf{r})$ that, in our quantum treatment, are found to be essentially insensitive to high-order binding potential effects over a wide interval of laser intensities. Thus, plateau structures in HHG spectra repeat those in the spectrum of odd QES harmonics of the KA wavefunction, i.e., they are in fact largely insensitive to the shape of $U(\mathbf{r})$ (at least, for finite-range potentials). Since the KA wavefunction describes the "free" evolution of a bound state $\psi_{0}(\mathbf{r}) \exp \left[-(\mathrm{i} / \hbar) E_{0} t\right]$ in a laser field, without any further interaction of the electron with the potential $U(\mathbf{r})$, it is not surprising that the plateau cut-off energy in HHG spectra is simply proportional to the classical energy $\mathcal{E}_{\mathrm{cl}} \approx 3.17 u_{p}$, which relates to freeelectron motion in the laser field.

Our quantum analysis for the TDER model is essentially exact and applicable over a wide interval of laser parameters. In particular, our results show that plateau features are typical not only for the low-frequency domain, $\hbar \omega \ll\left|E_{0}\right|$ (for which most of the quasiclassical analyses of ATI and HHG have been performed), but also for photon energies comparable to the binding energy, $\hbar \omega<\left|E_{0}\right|$ (see, e.g., the results for $\hbar \omega=0.46\left|E_{0}\right|$ in Figures 4 and 5, and in [22]). Also, our results demonstrate an evolution of ATD and HHG spectra with increasing laser intensity from the manifestly perturbative regime (when the lowest order PT results are applicable) to the strong field (non-perturbative) one, in which plateau structures become well developed. Although TDER results are most appropriate for systems involving weakly bound electrons, such as negative ions, we believe some of these results should also be valid for long-range (e.g., Coulomb) potentials. Indeed, the parity relation (22) is general and thus one may expect that the different sensitivity of plateau features in ATI and HHG to the shape of $U(\mathbf{r})$ should be valid in general. In particular, sophisticated Coulomb calculations of HHG spectra confirm the $3.17 u_{p}$-law, which does not depend on the details of the potential $U(\mathbf{r})$. 
Nevertheless, an analysis (presumably, numerical) of the spectrum of QES harmonics for the QQES wavefunction of an electron in a Coulomb potential $U(\mathbf{r})$ would be very desirable owing to the possibility of obtaining a better and more complete understanding of plateau features in intense laser-atom phenomena.

\section{Acknowledgments}

This work was supported in part by RFBR Grant 04-02-16350 and by NSF Grant PHY0300665. MVF acknowledges the support of the Dynasty Foundation and the Grant of the President of the Russian Federation MK-1075.2005.2.

\section{References}

[1] Kuchiev M Yu 1987 Zh. Eksp. Teor. Fiz. Pis. Red. 45319 Kuchiev M Yu 1987 JETP Lett. 45404 (Engl. Transl.)

[2] Schafer K J, Yang B, DiMauro L F and Kulander K C 1993 Phys. Rev. Lett. 701599

[3] Corkum P B 1993 Phys. Rev. Lett. 711994

[4] Becker W, Grabson F, Kopold R, Milošević D B, Paulus G G and Walther H 2002 Adv. At. Mol. Opt. Phys. $\mathbf{4 8} 35$

[5] Krause J L, Schafer K J and Kulander K C 1992 Phys. Rev. Lett. 683535

[6] Bauer D 2005 Phys. Rev. Lett. 94113001

[7] Mese E and Potvliege R M 2006 J. Phys. B: At. Mol. Opt. Phys. 39431

[8] Reiss H R 1980 Phys. Rev. A 221786

[9] Lohr A, Kleber M, Kopold R and Becker W 1997 Phys. Rev. A 55 R4003

[10] Manakov N L, Ovsiannikov V D and Rapoport L P 1986 Phys. Rep. 141319

[11] Potvliege R M and Shakeshaft R 1992 Atoms in Intense Laser Fields ed M Gavrila (New York: Academic) p 373

[12] Joachain C J, Dörr M and Kylstra N 2000 Adv. At. Mol. Opt. Phys. 42225

[13] Manakov N L and Rapoport L P 1975 Zh. Eksp. Teor. Fiz. 69842 Manakov N L and Rapoport L P 1975 Sov. Phys._JETP 42430 (Engl. Transl.) Berson I J 1975 J. Phys. B: At. Mol. Phys. 83078

[14] Manakov N L and Fainshtein A G 1980 Zh. Eksp. Teor. Fiz. 79751 Manakov N L and Fainshtein A G 1980 Sov. Phys.-JETP 52382 (Engl. Transl.)

[15] Demkov Yu N and Ostrovsky V N 1988 Zero-Range Potentials and Their Applications in Atomic Physics (New York: Plenum)

[16] Manakov N L, Frolov M V, Starace A F and Fabrikant I I 2000 J. Phys. B: At. Mol. Opt. Phys. 33 R141

[17] Manakov N L, Frolov M V, Borca B and Starace A F 2003 J. Phys. B: At. Mol. Opt. Phys. 36 R49

[18] Manakov N L, Starace A F, Flegel A V and Frolov M V 2002 Zh. Eksp. Teor. Fiz. Pis. Red. 76316 Manakov N L, Starace A F, Flegel A V and Frolov M V 1987 JETP Lett. 76258 (Engl. Transl.) Flegel A V, Frolov M V, Manakov N L and Starace A F 2005 Phys. Lett. A 334197

[19] Flegel A V, Frolov M V, Manakov N L and Starace A F 2005 J. Phys. B: At. Mol. Opt. Phys. 38 L27

[20] Frolov M V, Manakov N L, Pronin E A and Starace A F 2003 Phys. Rev. Lett. 91053003

[21] Frolov M V, Manakov N L, Pronin E A and Starace A F 2003 J. Phys. B: At. Mol. Opt. Phys. 36 L419

[22] Frolov M V, Flegel A V, Manakov N L and Starace A F 2005 J. Phys. B: At. Mol. Opt. Phys. 38 L375 
[23] Landau L D and Lifshitz E M 1978 Quantum Mechanics 2nd edn (Oxford: Pergamon)

[24] Keldysh L V 1964 Zh. Eksp. Teor. Fiz. 471945 Keldysh L V 1965 Sov. Phys.—JETP 201307 (Engl. Transl.)

[25] Faisal F H M 1973 J. Phys. B: At. Mol. Phys. 6 L89

[26] Becker A and Faisal F H M 2005 J. Phys. B: At. Mol. Opt. Phys. 38 R56

[27] Lewenstein M, Kulander K C, Schafer K J and Bucksbaum P H 1995 Phys. Rev. A 511495

[28] Lewenstein M, Balcou Ph, Ivanov M Yu, L'Huillier A and Corkum P B 1994 Phys. Rev. A 49 2117

[29] Kuchiev M Yu and Ostrovsky V N 1999 Phys. Rev. A 603111

[30] Langhoff P W, Epstein S T and Karplus M 1972 Rev. Mod. Phys. 44602

[31] Gell-Mann M and Low F 1951 Phys. Rev. 84350

[32] Sucher J 1957 Phys. Rev. 1071448

[33] Mohr P J, Plunien G and Soff G 1998 Phys. Rep. 293227

[34] Zel'dovich Ya B 1966 Zh. Eksp. Teor. Fiz. 511492 Zel'dovich Ya B 1967 Sov. Phys._JETP 241006 (Engl. Transl.)

[35] Manakov N L and Fainshtein A G 1981 Teor. Mat. Fiz. 48375 Manakov N L and Fainshtein A G 1982 Theor. Math. Phys. 48815 (Engl. Transl.)

[36] Potvliege R M and Shakeshaft R 1989 Phys. Rev. A 403061

[37] Potvlige R M and Shakeshaft R 1988 Phys. Rev. A 386190

[38] Baz' A I, Zel'dovich Ya B and Perelomov A M 1971 Scattering, Reactions and Decays in Nonrelativistic Quantum Mechanics 2nd edn (Moscow: Nauka)

[39] Nikishov A I and Ritus V I 1966 Zh. Eksp. Teor. Fiz. 50255 Nikishov A I and Ritus V I 1967 Sov. Phys.-JETP 23168 (Engl. Transl.)

[40] Bauer D, Becker W and Milošević D B 2005 Phys. Rev. A 72023415

[41] Becker W, Long S and McIver J K 1994 Phys. Rev. A 501540

[42] Borca B, Flegel A V, Frolov M V, Manakov N L and Starace A F 2002 Phys. Rev. A 65051402

[43] Fainshtein A G, Manakov N L, Ovsiannikov V D and Rapoport L P 1992 Phys. Rep. 210111 